\title{
Shape Description and Matching Using Integral Invariants on Eccentricity Transformed Images
}

\author{
Faraz Janan • Michael Brady
}

Received: 23 September 2013 / Accepted: 4 October 2014

(C) Springer Science+Business Media New York 2014

\begin{abstract}
Matching occluded and noisy shapes is a problem frequently encountered in medical image analysis and more generally in computer vision. To keep track of changes inside the breast, for example, it is important for a computer aided detection system to establish correspondences between regions of interest. Shape transformations, computed both with integral invariants (II) and with geodesic distance, yield signatures that are invariant to isometric deformations, such as bending and articulations. Integral invariants describe the boundaries of planar shapes. However, they provide no information about where a particular feature lies on the boundary with regard to the overall shape structure. Conversely, eccentricity transforms (Ecc) can match shapes by signatures of geodesic distance histograms based on information from inside the shape; but they ignore the boundary information. We describe a method that combines the boundary signature of a shape obtained from II and structural information from the Ecc to yield results that improve on them separately.
\end{abstract}

Keywords Shape matching - Shape correspondence . Integral invariant - Eccentricity transform · Fast Marching Algorithm $\cdot$ CAD $\cdot$ Breast cancer

Communicated by Gerard Medioni.

F. Janan ( $($ )

Biomedical Engineering, University of Oxford, Oxford, UK

e-mail: faraz.janan@some.ox.ac.uk; farazjanan@yahoo.com

F. Janan

Matakina UK Limited, Cambridgeshire, UK

M. Brady

Oncology, University of Oxford, Oxford, UK

e-mail: mike.brady@oncology.ox.ac.uk

\section{Introduction}

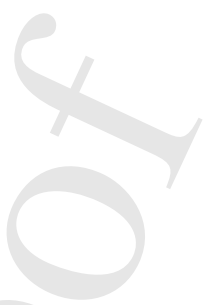

Shape matching and finding a suitable set of correspondences an important computer vision problem that has received considerable attention, particularly over the past few years. Shape transformations, computed both with integral invariants (II) and geodesic distances yield signatures that are invariant to isometric deformations, such as bending and articulations. Geometric invariant functions are generally used to describe the shapes that result from images taken under various transformations such as affine, similarity, Euclidean, or a range of projection types. Shape matching applications, for example in medical image analysis, necessitates shape description. If we consider images of two similar objects, or of the same object taken at different times, angles, and from varying distances, we expect to find changes in the extracted shapes. Of course, the use of projective invariants can cope with many such changes. However, shape variations may be in the form of missing data, with complete or partial articulations, and in many practical applications, particularly in medicine and biology, such changes are significant. For example, in oncology, such changes may indicate regions of new growth. Past efforts to compare two shapes have typically involved image registration techniques, for example (Mardia and Dryden 1989; Mumford 1991).

However, most such approaches depend upon a 'shape space' and require (often extensive) training data before actual comparisons are possible (Kendall 1984). Most published algorithms that are based either on rigid or nonrigid image registration typically yield a dense warp map, establishing correspondences for all pixels in the shapes. Typically, they focus on shape matching rather than localizing matching to regions. Indeed, there appears to have been little or no research aimed at identifying and quantifying new growths and partial occlusions by comparing 
Fig. 1 List of shape analysis method and their classification (mostly described in Zhang and Lu 2004) two planar shapes regardless of scale, spatial variations, and orientation.

We are particularly interested in describing and comparing two planar contours with no self-intersections in a two dimensional space. Shape descriptors can be used to find point-wise correspondences typically in terms of extremizing a shape distance or matching cost between the two shapes. Our main interest is in descriptors that define edges, corners, peaks and ridges. The sensitivity of differential measures to small perturbations due to noise limits their use in shape matching and generally does not produce adequate results at increasing scale.

We have used (circular) II to describe the boundaries of shapes. This creates a scale space in which the integral invariant defines features for the shape at a range of scales. The particular application on which we focus is mammographic analysis: matching potential masses (benign or malignant). Though II have been used to describe the shape boundary, they provide no information about where a particular feature on the boundary lies with regard to the overall shape structure. Conversely, eccentricity transforms (Ecc) can be used to match shapes by signatures of geodesic distance histograms based on information from inside the shape; but they ignore the boundary information. In this paper, we describe a method that combines the boundary signature of a shape obtained from II and structural information from the Ecc to yield results that improve on them separately.

\section{Background}

As our method involves the use of II, the Ecc, and the Fast Marching Algorithm (FMA) for shape matching and estab-

\section{Shape Descrition}

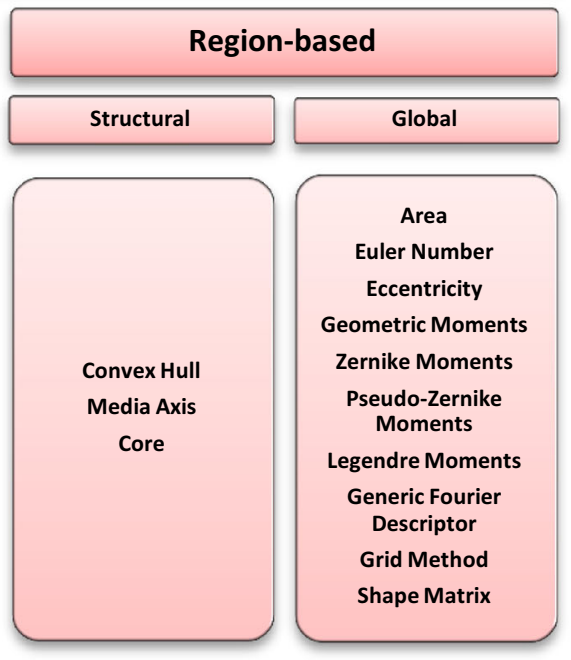

lishing correspondences, the spectrum of existing related work is naturally extensive. A comprehensive review is provided in the first author's PhD thesis; here, we must necessarily be selective.

\section{Shape Representation}

We assume that the shapes of interest assume the form of closed 2-D planar contours. We further assume that the shape describes a single entity that has a geometrical pattern (Xu 2008), which persists modulo some suitable transformation group (Amanatiadis et al. 2011; Duci et al. 2003; Kendall 1984; Mardia and Dryden 1989; Mumford 1991; Sharon and Mumford 2006; Zhang and Lu 2004). Mathematically, shapes are described in the form of descriptors that are ideally invariant to scale, rotation, translation, and, where appropriate, reflection. Such descriptors are applied at several scales, in order to make explicit anatomical structures at different levels of observation. A detailed review of shape representation and description techniques along with their categorical classification is given in Amanatiadis et al. (2011) and Zhang and Lu (2004). Figure 1 shows one classification of current methods for shape description.

Several contour-based methods have been reported. Duci et al. (2003) suggested embedded closed planar contours that possess a linear signature as a subset of harmonic functions of which the original contour is a zero level-set. Sharon and Mumford (2006) generated a series of conformal maps, starting from mapping the object to a unit circle in the complex plane, then from the boundary of the object to the exterior of the circle, so that the final boundary is a diffeomorphism 


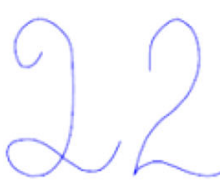

(a)

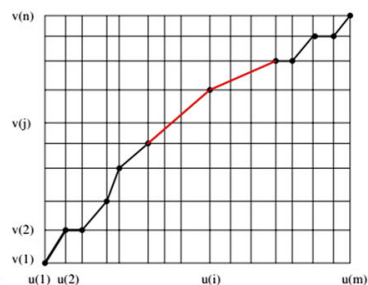

(b)

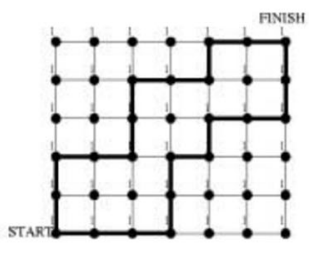

(c)

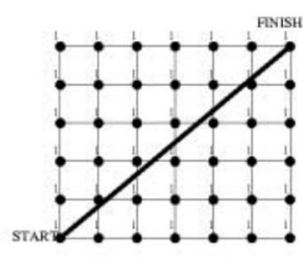

(d)

Fig. 2 a An example of the number 2 drawn in different ways and illustrating two curves that are to be put in correspondence. b An example of the discrete alignment curve in (a) given as a shortest path in a

from the unit circle to itself. They call this the finger print of the shape. B-Splines are widely used for shape representation and curve matching (Cohen and Wang 1994; Gu and Tjahjadi 2000; Huang and Cohen 1996; Wang et al. 2004, 2001; Wang and Teoh 2004). Biological sequence dynamic alignment (Zhang and Ma 2000) and Chain codes have also been used for contour based shape representation (Yu et al. 2010; Zhang and Ma 2000; Arrebola and Sandoval 2005) though they are not considered reliable for shape matching, mainly because they suffer from discretization errors with respect to rotation and scale (Fig. 2). Typically, in shape matching applications, curvature functions of the contour are used to encode the boundary of an object (Mokhtarian et al. 1997; Mokhtarian and Mackworth 1986). Such a differential representation is attractive because it represents an object in a well investigated mathematical framework (Olver 1995; Thomas 1934; Amanatiadis et al. 2011). However, a major practical shortcoming of differential invariants is that they are based on derivatives which are sensitive to noise and small perturbations. The global behaviour of differential invariants reduces its robustness to noise. It is known that any orders of differential invariants on a plane are functions of curvature (Weiss 1993).

In contrast to this, Maney et al. (2004) used Integral Invariants to describe shapes with similar invariant properties as their differential counterparts. This approach has been used for shape reconstruction (Huang et al. 2006) and found to be more robust to noise (Manay et al. 2006, 2004; Yang et al. 2006). Sato and Cipolla (Sato and Cipolla 1997, 1996) showed that II out-perform differential invariants because of their lower noise sensitivity. Recently, it has been shown that circular II provide a unique representation for each shape (Bauer et al. 2011), as do conic II (Huang et al. 2006). Integral Invariants may be viewed as a structural approach since they represent a shape in terms of boundary primitives.

An advantage of such a structural invariant approach is the ability to handle occlusions and possibility of partial matching in shapes. These are of considerable importance in medical imaging. graph. c Multiple "shortest" network paths, showing how Dynamic programming suffers from the city block distance problem. $\mathbf{d}$ The optimal diagonal path is the result of the FMA (Frenkel and Basri 2003)

More recently, Ecc (Ion et al. 2011, 2008, 2007), Bending invariants (Elad and Kimmel 2003; Rosin 2011) and Skeletonization (Sundar et al. 2003; Xu et al. 2010) of shapes have been applied with success for shape description and retrieval. They take advantage of the information from inside of a shape. In this paper we use the Ecc because of its undistorted representation of a shape.

\subsection{Shape Invariants}

Usually, invariants refer to properties that remain unchanged under an appropriate class of transformations (such as similarity transformations) (Sonka et al. 1999). Transformations collectively form a group, such as the projective groups used widely in computer vision, because transformations can be composed and inverted. Such groups provide mathematical tools (group actions) for generating invariants that are applied to a range of applications (Alferez and Wang 1999; Belongie et al. 2002; Bengtsson and Eklundh 1991; Brandt and Lin 1996; Bruckstein et al. 1997; Chetverikov and Khenokh 1999; Cohignac et al. 1994; Li 1999; Mumford et al. 1984; Reiss 1993) and are considered to be the basis of invariant theory (Amanatiadis et al. 2011; Helgason 1984). Invariants are described by the number of features that define their order.

A broad review of different type of invariants used for shape description for the purposes of matching is given in $\mathrm{Li}$ (1999). The four most common types of invariants are:

(1) Algebraic invariants (Forsyth et al. 1990; Nielsen and Sparr 1991; Sonka et al. 1999; Squire and Caelli 2000) such as Eigenvalues, trace, and determinant. Algebraic invariants additionally require the correspondence of distinguished points to establish matching of two shapes.

(2) Geometric invariants (Huang and Huang 1998; Li 1999) such as distance transforms, measurement ratios, and invariants computed from a combination of coplanar points or planes (Forsyth et al. 1991, 1990; Gool et al. 1996; Lasenby et al. 1996; Nielsen and Sparr 1991; Roth- 
well et al. 1995, 1992; Shashua and Navab 1996; Zisserman et al. 1995)

(3) Differential invariants that are essentially invariant to Lie group actions, such as torsion, Gaussian measures and curvature (Belongie et al. 2002; Calabi et al. 1998; Cao et al. 2011; Cole et al. 1991; Kanatani 1990; Lenz 1990; Olver 1995; Trucco 1995; White et al. 2004). Differential invariants do not require correspondence of image features; however, they are based on higher order derivatives that make them sensitive to noise.

(4) Integral invariant such as semi-local affine (Sato and Cipolla 1996), integral moments (Taubin and Cooper 1991), circular (Manay et al. 2004) and conic invariants (Fidler et al. 2007).

Also, almost all invariants of types (1-3) are sensitive to boundary noise. However, II are comparatively robust to noise. Circular II are similar to the SUSAN feature detector (Smith and Brady 1997) which has been used in a range applications (Arun and Sarath 2011; Zhou et al. 2011; Mansoory et al. 2011; Rezai-Rad and Aghababaie 2006; Si-ming et al. 2011; Xu et al. 2006; Zeng and Li 2011) and reproduced with various enhancements (Qu et al. 2011; Rafajlowicz 2007; Xingfang et al. 2010; Zeng and Li 2011). One of the major limitations of the SUSAN method for our application is that it assumes that the pixels which belong to a circular region are homogeneous (i.e. have relatively uniform brightness), which is generally not the case in medical images, which are generally piecewise homogeneous. One major issue with invariants when they are used for shape matching is that they have to be formulated as the intrinsically NP-complete problem ( $\mathrm{Li}$ 1992) of finding the relationship between parts of shapes and of establishing a one-to-one correspondence for producing a matching cost. This reduces the problem to searching for an acceptable match rather than a definite solution given in a reasonable time. To deal with this problem and to partially reduce the computational cost, shape signatures have been proposed (Bauer et al. 2011; Davies 2004; Fidler et al. 2007; Kliot and Rivlin 1998; Manay et al. 2004; Squire and Caelli 2000).

\subsection{Shape Matching}

Shapes are usually matched by establishing correspondences on points along the boundaries of the two shapes. Shape matching either uses the intrinsic statistical properties of the shapes or by anatomical modelling and then by corresponding the boundary points to compute a matching cost, a standard practice in shape retrieval (Gdalyahu and Weinshall 1999; Petrakis et al. 2002). This is done by identifying salient landmarks (Davis 1977) using various shape descriptors, such as variational methods (Veltkamp 2001), phase information(Belongie et al. 2002; Rusinol et al. 2007), Eccentric- ity (Ion et al. 2007), genetic algorithms (Ozcan and Mohan 1997) and curvature (Mokhtarian et al. 1997; Mokhtarian and Mackworth 1986, 1992).

Shape matching depends on the type of descriptor used (Mardia and Dryden 1989). Transformation-based descriptors, such as Fourier components (Zahn and Roskies 1972), which amplify certain features of a shape, usually suppress other important information such as local deformations, translation and rotation (Ion et al. 2007). Shape matching that aims to find dense correspondences is particularly challenging in the case of articulated shapes. Such correspondence techniques (Belongie et al. 2002; Bronstein et al. 2006; Mateus et al. 2008; Ruggeri et al. 2010; Sharma and Horaud 2010; Wang et al. 2007) embed 2D or 3D shapes in a canonical domain that largely preserves geodesic distances (Bronstein et al. 2008; Hamza and Krim 2006; Osada et al. 2002), angles, and other important properties of the structure, and leads to isometric deformations, such as bending (Ling and Jacobs 2007; Nasreddine et al. 2009) and articulations. Other techniques involve feature analysis based on graph matching (Duchenne et al. 2011; Leordeanu and Hebert 2005; Maciel and Costeira 2003; Torresani et al. 2008), which also combines the appearance of shapes. Laplace spectra (Reuter et al. 2005), contour flexibility (Xu et al. 2009), shape skeletons (Siddiqi et al. 1999), the rolling penetrate descriptor (Chen and Xu 2009), and partial differential equations (Gorelick et al. 2006) have been explored. Shape correspondence using histogram geometry for $2 \mathrm{D}$ shapes, which has also been extended to 3D, decomposes shapes into parts using topographic features and eventually registers them (Reuter et al. 2005). Recent evolutionary shape matching techniques, such as Ant Colony Optimization (ACO) (Van Kaick et al. 2007; Tian et al. 2011a, b), Bee Colony Optimization (BCO) (Davidovic et al. 2010; Shi et al. 2013; Teodorovic et al. 2011) and Artificial Bee Colony (ABC) (Xu and Duan 2010) have aroused interest among the shape analysis community. Comprehensive surveys of shape matching techniques with respect to correspondence can be found in Van Kaick et al. (2011), Mikolajczyk and Schmid (2005) and Veltkamp and Hagedoorn (2001).

Sebastian (2003) proposed a novel approach to curve correspondence based on alignment criteria with respect to a model curve. The optimal correspondence problem was addressed using Dijkstra's algorithm (Dijkstra 1976, 1968, 1959), which solves the functional equation for the shortest path problem using dynamic programming. The algorithm was tested on the retrieval of 1,400 shapes belonging to 70 different categories each consisting of 20 shapes. The percentage of correct correspondences was $78.17 \%$, which was claimed to be the best published retrieval rate when compared to: curvature scale space (Mokhtarian et al. 1997); comparison using visual parts (Latecki et al. 2000); and shape contexts (Belongie et al. 2001) which give 75.44, 76.45 and 
$76.51 \%$ respectively. However, one of the limitations of this approach is that it cannot deal with flipped shapes and, more seriously, it suffers from the initial alignment problem. Optimal alignment for each pair of shapes is found before and after flipping the shape and the one with the lowest cost is proposed. However, this multiplies the computational cost of the algorithm. Both the Manay et al. (2006) and Sebastian et al. $(2003 ; 2001)$ algorithms to find correspondences between shapes use Dynamic programming based on Dijkstra's algorithm. However, this algorithm suffers from sub-pixel accuracy and the city block distance problem in finding the shortest path to establish point-wise correspondences. To address this problem, we use the FMS (Kimmel 2004; Sethian 1999).

\section{Methods}

\subsection{Circular Integral Invariant}

Manay et al. (2006) used circular II for shape matching. These are invariant under a group of transformations and suitable for use when the shape is occluded. We are interested in local circular area II for their simplicity, robust shape description, and properties of non-emergence and non-enhancement of extrema in feature space at varying scales. At various points in this paper circular integral invariants are used for noise suppression, shape matching, and region matching with a multi-scale representation. They resemble a Gaussian kernel in implementation; however, they differ substantially in their diffusion properties.

An Integral Invariant is defined (Manay et al. 2006) by considering a disc $B_{r}(p)$ of radius $r$ applied to every point $p$ of a closed contour $C$. The characteristic function is then given by,

$\chi\left(B_{r}(p), C\right)(x)= \begin{cases}1 & \text { if } x \in\left\{B_{r}(p) \cap \dot{C}\right\} \\ 0 & \text { otherwise }\end{cases}$

where $\dot{C}$ is the interior of the curve $C$. The local integral area $I_{r}(C)$ of the curve $\mathrm{C}$ is given by the function $I_{r}(p)$ at every point $p \in C$ with integral kernel $\chi$ as follows:

$I_{r}(p)=\int_{\Omega} \chi\left(B_{r}(p), C\right)(x) d x$

where $\Omega$ is the domain of the curve $C$. Figure 3 illustrates II as per (Manay et al. 2004) and Eq. 2. The size of the integral kernel $r$ can be varied, yielding a scale space, without worrying about amplification of noise. In fact, results show that by increasing the range and scale of integration, the kernel suppresses noise and gives more robust results; however it adversely affects the shape details. The value of the Integral Invariant for shape description is if the circle is centred not on

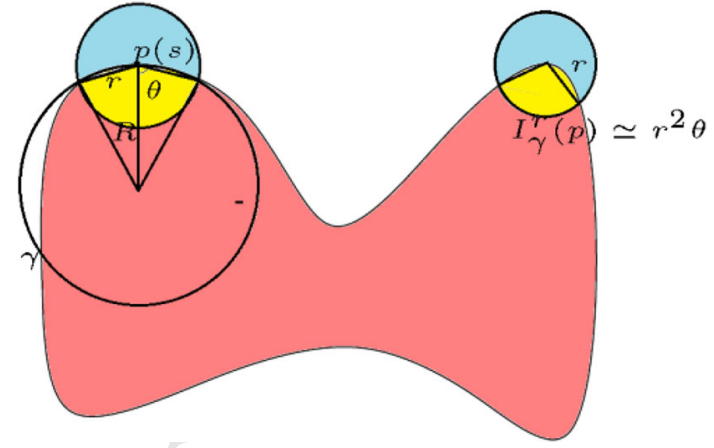

Fig. 3 Area integral invariant defined in Eq. 2

a point along the curve but near to it, so that the circle overlaps the shape interior. Two examples of integral invariant shape description are shown in Fig. 4.

It will be shown that II have strong expressive power to encode a shape and that it is closely related to representations using curvature. In fact, in a certain sense, it is a weighted reciprocal of curvature. The maxima of integral invariant are the minima of curvature; but they have far greater resistance to noise. A problem with II is scale selection. There is a certain ratio of the size of the shape and integration kernel that has to be maintained. The size of the kernel should be small enough to make explicit localized changes, yet large enough to establish the global position of a shape region in an image. As the size of the kernel is increased, its sensitivity to noise decreases. Results show that, compared to differential invariants, integral invariants are robust to noise and are that they are effective for shape correspondence. A detailed mathematical comparison of projective curvature and II is given in Hann and Hickman (2002) and with applications in Pottmann et al. (2009).

Circular II can also be obtained by the differentiation of area invariants as given in Bronstein et al. (2008). Figure 4 demonstrates the application of II to two example shapes.

\subsection{Eccentricity Transform}

Eccentricity transforms are also robust to noise (Ion et al. 2011). Ecc determines the geodesic distance for each point within a shape, to every other point on the boundary. Figure 5 illustrates the geodesic as compared to Euclidean distance inside a shape. It then assigns to each point a distance to the point farthest away from it. Instead of assigning the maximum distance, the mean, median or minimum distance may also be used as shown in Fig. 6.

The geodesic distances are calculated using the Fast Marching Algorithm (FMA). The Ecc shape matching algorithm, matches histograms obtained from Ecc transformed images. Such a geodesic distance histogram does not explicitly contain boundary information, including information 
(a)
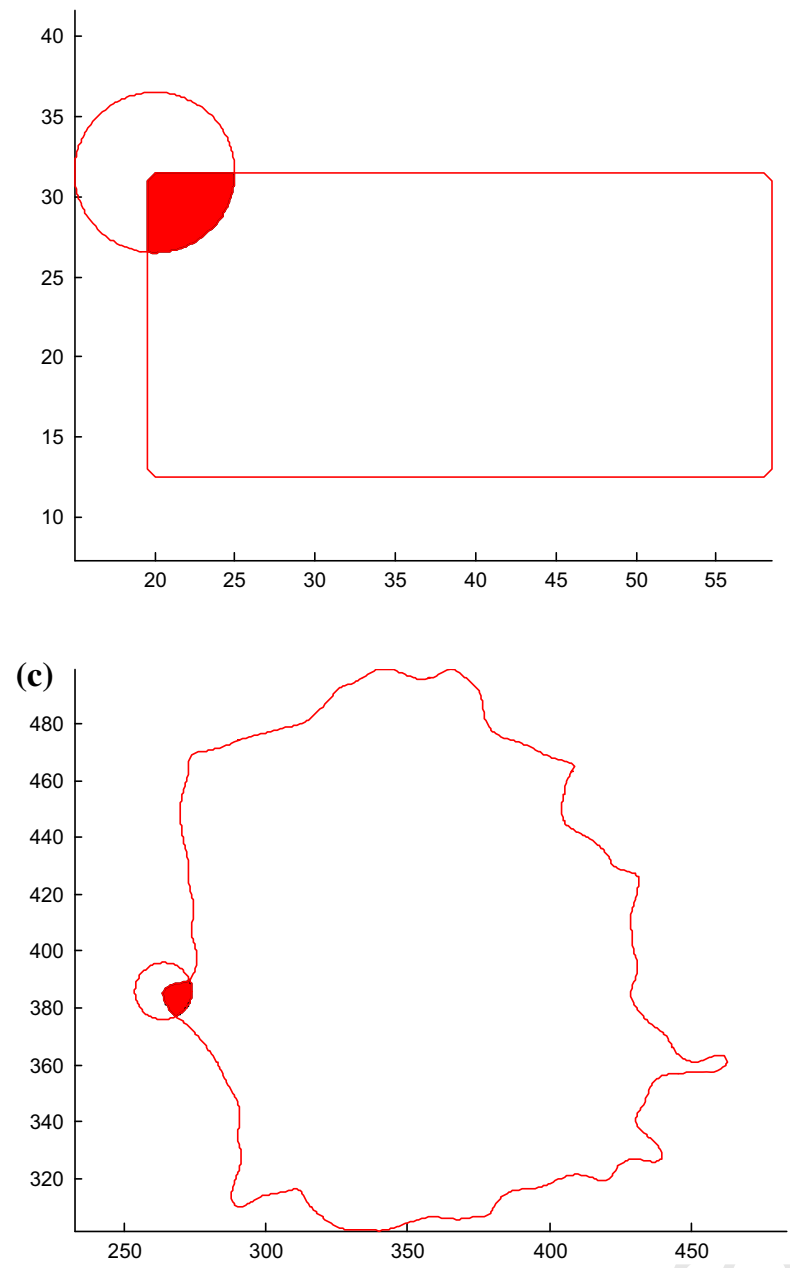

Fig. 4 a, c are two examples of closed polygons with integration kernels imposed on them and highlighting the integration area in red, $\mathbf{b}$, d are the corresponding Integral Invariant for the complete curves. c (b)
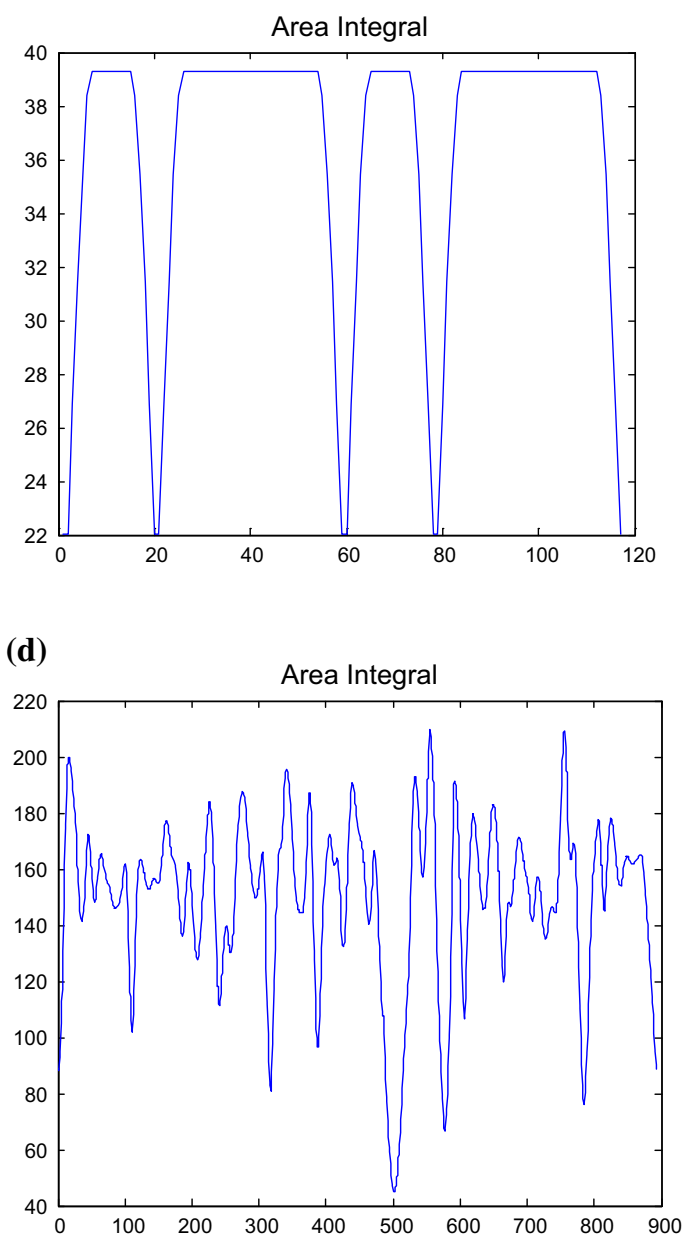

is the outline of a segmented mass in mdb010 from the Mini-MIAS mammographic database (Janan and Brady 2012) (Color figure online)

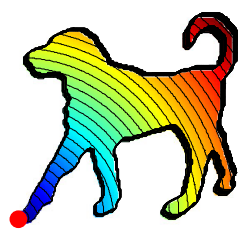

(a)

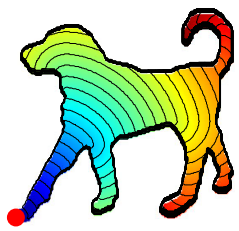

(b)

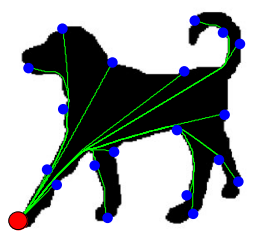

(c)
Fig. 5 A Euclidean and geodesic space representation. a Euclidean distance shown in level contours inside the shape from the red dot in the bottom-left end of the shape. b Geodesic counterpart of the shape on the left, which is attained using the Fast Marching Algorithm (FMA). c Illustration of geodesic paths to various points on the shape boundary such as curvature. They do not appear to have been used previously for establishing point-wise shape correspondences between shapes.
Adrian (Ion et al. 2007) defines the Ecc by considering a shape $S \subset \mathrm{R}^{2}$ with a smooth boundary $\partial S$, where $S$ may be an image $f_{s}$ of $n * m$ pixels, such that,

$f_{s}(x)= \begin{cases}1 & \text { for } x \in S \\ 0 & \text { otherwise }\end{cases}$

The geodesic distance $d_{s}(x, y)$, between any two points $x$ and $y$ on the shape $\mathrm{S}$ is given by,

$$
d_{s}(x, y) \stackrel{\text { def }}{=} \min _{\gamma \in p(x, y)} L(\gamma)
$$$$
\text { where } L(\gamma) \stackrel{\text { def }}{=} \int_{0}^{1}\left|\gamma^{\prime},(t)\right| d t
$$

where, $p(x, y)$ is the set of paths $\gamma(t)$ from $x$ to $y$, such that 


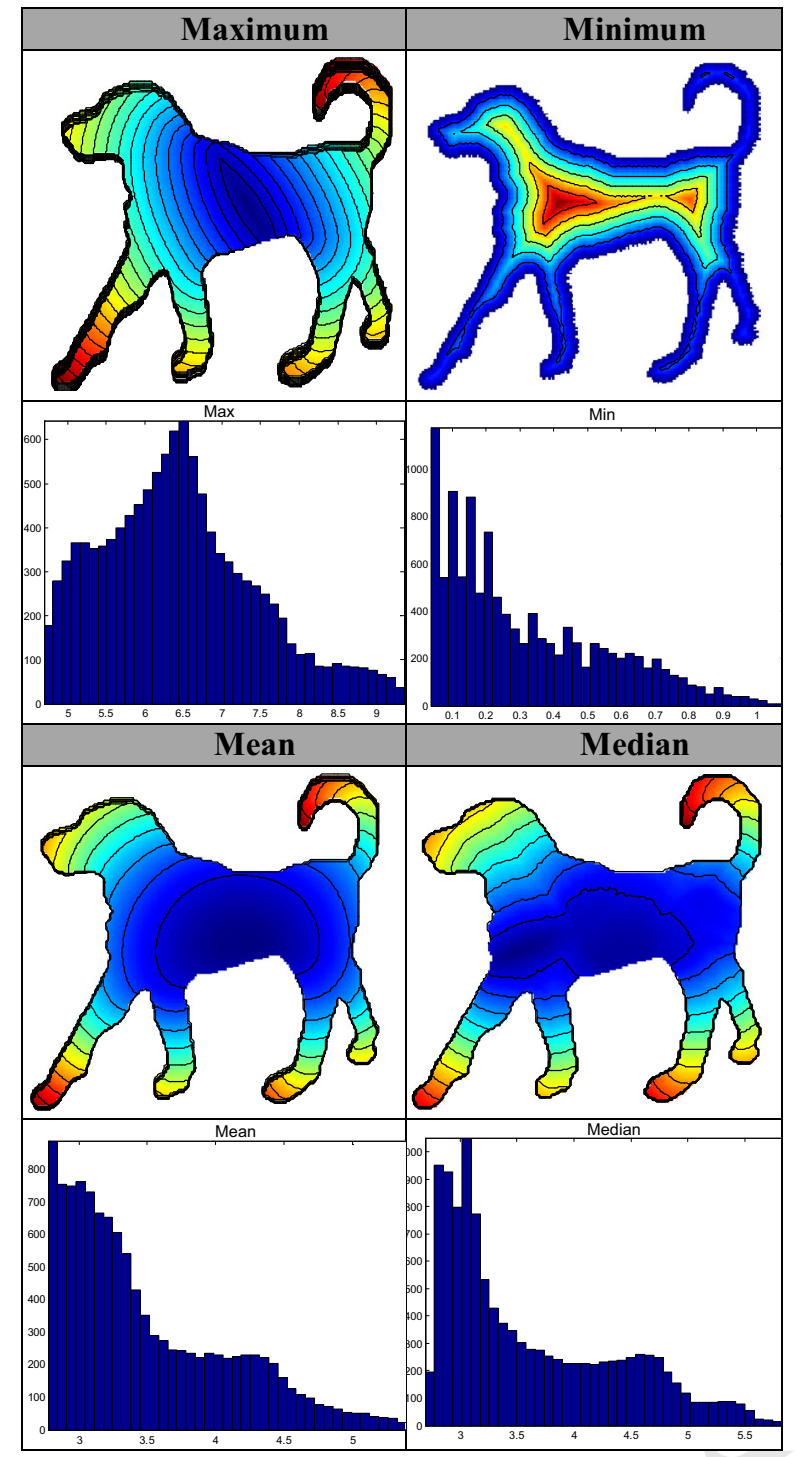

Fig. 6 Eccentricity transformed shapes and their corresponding histograms underneath each shape. The top column shows what type of distance is taken into account from the feature space while finding shape transformations

$y(t)=x, \quad$ for $t=0$

$y(t)=y, \quad$ for $t=1$

Inside the shape, and for any starting point $x_{0}$, the distance function $U(x) \stackrel{\text { def }}{=} d\left(x_{0}, x\right)$ can be computed by the finding the solution to the Eikonal equation,

$\forall x \in S, \nabla U(x)=1$, and $U(0)=0$

The FMA is used to solve the above Eikonal equation to find the minimum path between $x_{0}$ and $x$.

The Ecc of $S$ to each point $p \in S$ is the shortest geodesic distance to the point on $S$, farthest away from it.
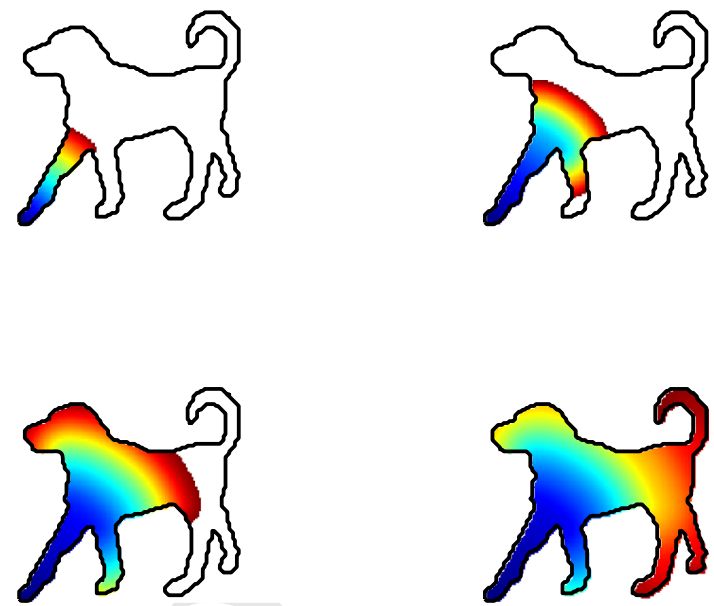

Fig. 7 Illustration of Fast Marching Algorithm exploring an animal shape

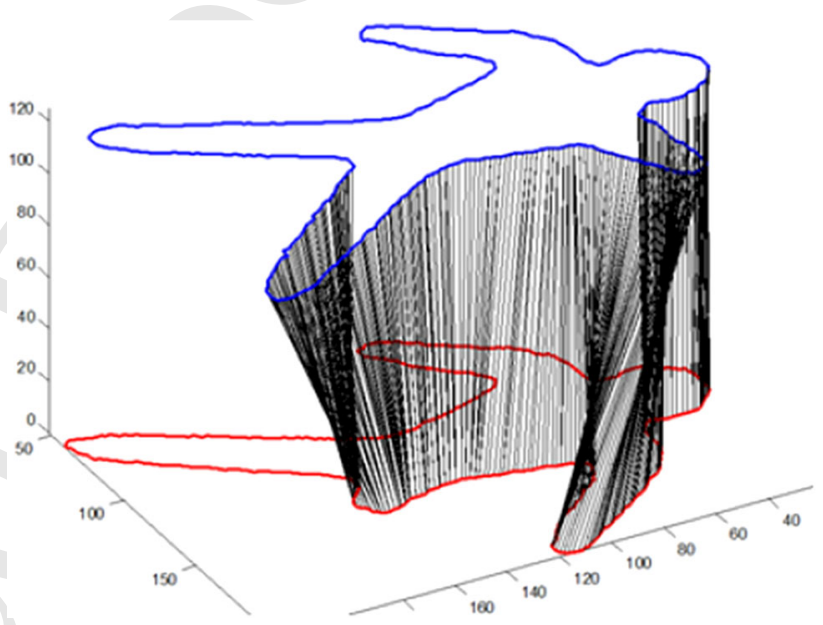

Fig. 8 Two shapes corresponded using Integral Invariant and Fast Marching Algorithm

The feature set is calculated as follows: the distance for each point inside the shape is calculated to every point in the boundary, thus forming $I_{n} \times I_{m} \times n$ feature space, where $\mathrm{I}_{\mathrm{n}} \times \mathrm{I}_{\mathrm{m}}$ are the image dimensions and $\mathrm{n}$ is the parameterization of the boundary curve $\partial \mathrm{S}$.

$\operatorname{Ecc}_{S}(x) \stackrel{\text { def }}{=} \max _{y \in S} d_{S}(x, y)=\max _{y \in \partial S} d_{S}(x, y)$

394

395

396

397

398

The original paper (Ion et al. 2007) on Ecc shape matching calculates a histogram to calculate the shape signature, without giving boundary correspondences. We have used II to perform shape matching and to establish boundary correspondences.

\subsection{Fast Marching Algorithm}

The FMA computes the viscosity solution of the Eikonal equation. Assume a two dimensional real domain $\Omega$ where
406 407 


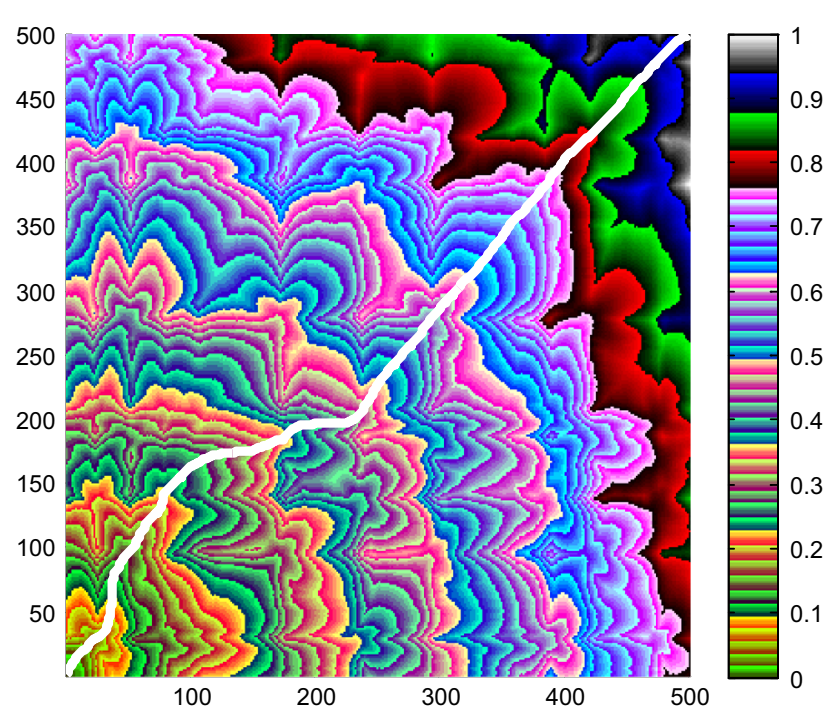

Fig. 9 Geodesic distance map calculated by the Fast Marching Algorithm for shapes corresponded in Fig. 5, along with the shortest path superimposed as a white line on the feature map, which follows the lowest values or matching cost

Fig. 10 Distance map created by FMA, where Geodesic path is being calculated using Gradient Descent, shown as a blue line passing across the diagonal (Color figure online) fire front may take a new direction with new adjacent and far points. Hence there are points that are burnt, points next to fire and points far from fire-yet to be explored. This process continues until all points are reached and explored. This simulation is the core idea underlying the FMA. The output of a Fast Marching Algorithm is a distance map starting from initial point to the final point, exploring all points in the map. (Fig. 7)

The FMA is computationally more efficient than previous attempts to solve the Eikonal equation (Helmsen et al. 1996; Tsitsiklis 1995), including: the Fast Sweeping Algorithm (Boué and Dupuis 1999; Tsai et al. 2003; Zhao 2005), and Dynamic Programing (Bertsekas 1995; Hadley 1964; Petrakis et al. 2002; Sniedovich 2010). FMA has been applied to active contours (Cohen and Kimmel 1997) and to shape from shading (Kimmel and Sethian 2001). Frenkel and Basri (2003) used FMA to solve the Eikonal equation to align handwriting shapes. It implements curvature information to match closed curves, morphs one curve into another, and can find the average curve for a group. Experiments were carried out on 110 shapes and the results of 13 experiments performed on the complete database were promising. The behaviour of open shapes with arms and teeth (numbers and alphabets) led to interesting conclusions about the relationship between curvature and shape correspondence. However, the method failed to quantify the difference between two shapes other than matching cost. In this paper, we develop a framework that can match, and then quantify, real shape differences.

\section{Implementation}

We match shapes in a two-step procedure. First, the Ecc is applied to each shape separately to define the spatial lay- 
Table 1 Establishing point-wise correspondence

\section{Algorithm}

1. Parameterize the boundaries of Shapes 1 and Shape 2 so that they both have $n$ points.

2. Select a set of $k$ scales and for each scale apply Integral Invariants to Shape 1 and Shape 2 at that scale; this results in $k$ signatures for each shape.

3. The Integral Invariant difference for comparing points $P 1$ and $P 2$ in Shape 1 and Shape 2 is computed at all $k$ scales, resulting in a difference vector. Compute the largest singular value by singular value decomposition of the $1 \mathrm{x} k$ difference vector. This is considered to be the largest feature difference between points $P 1$ and $P 2$. This process is continued until the largest singular value feature difference is found for every point in Shape 1 against every point in Shape 2

4. The result of this process is an $n \times n$ feature matrix, such that at each point in the matrix contains the largest feature difference for the two associated points in Shape 1 and Shape 2 at all given scales.

5. The Fast Marching Algorithm is applied to the $n \times n$ feature matrix, creating a distance map.

6. The geodesic distance along the diagonal of the distance map is found using gradient descent. This indicates the lowest match cost while mapping points on two shapes.

7. The geodesic path maps points of Shape 1 to points on Shape 2 and establish a point wise correspondence between the two shapes.

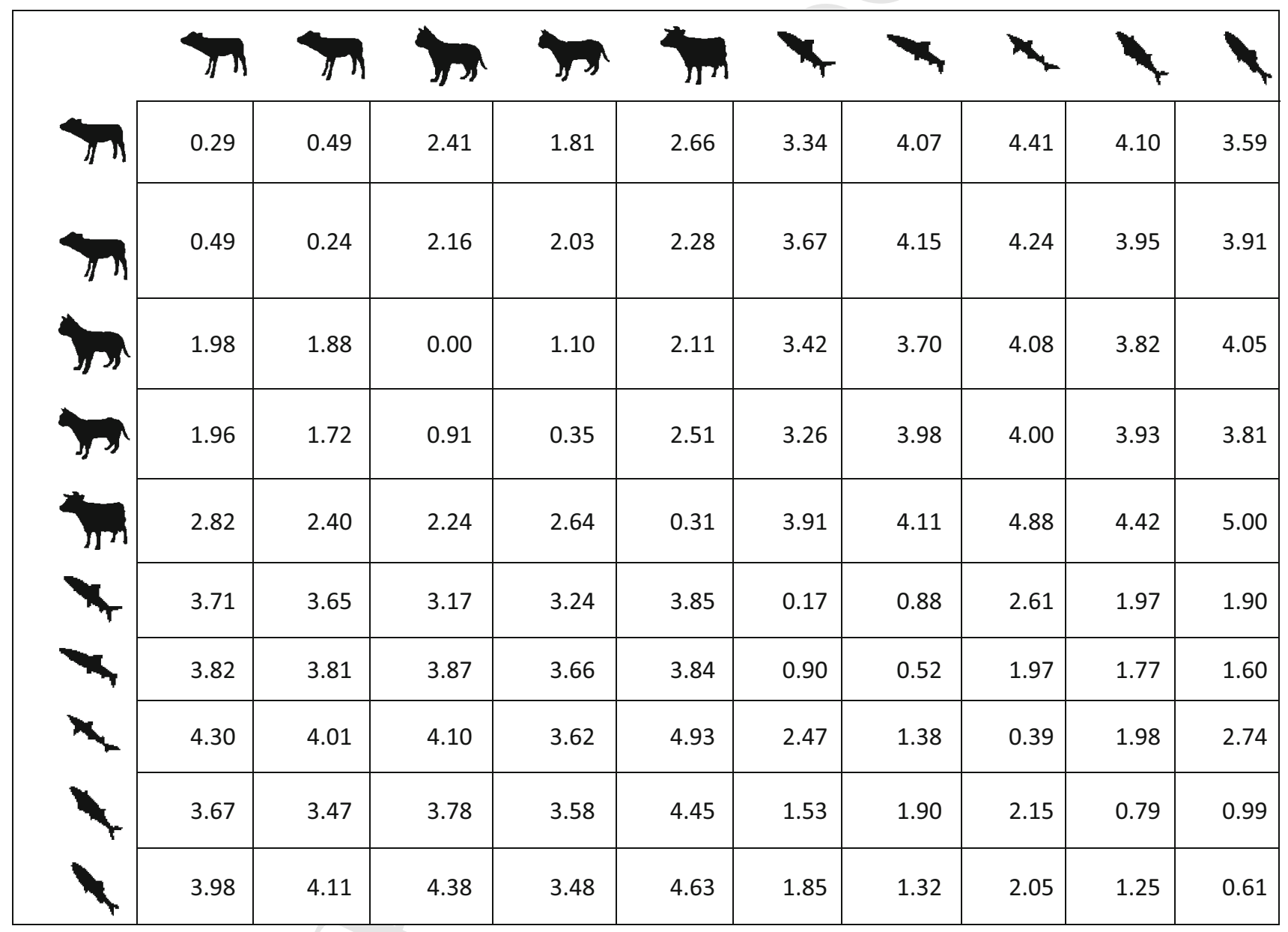

Fig. 11 Matching cost of the two shape families that are matched in the presence of noise using Fast Marching Algorithm. The differences are easily observed in Fig. 12 in a false coloring model

out of regions inside each of them. Second, pointwise correspondences of the boundary points are established using II, hence for regions inside the shapes, as well as generating a matching cost. We first describe the process of establishing pointwise correspondences, as it is also used subsequently to explain the viability of Ecc. 
Fig. 12 The matching cost is color coded. It can be seen that strong matching is observed (shown in blue) along the diagonal

\subsection{Pointwise Correspondence}

The following steps adapted from Peyré (2011) establish pointwise correspondences between Shape 1 and Shape 2.

The singular value for the difference vector, which is the Integral Invariant difference for a proposed correspondence at all scales, gives the distance of that point from the origin in feature space. For a three dimensional feature vector $x$, the singular value decomposition (svd) is, $x=\left[\begin{array}{lll}x_{1} & x_{2} & x_{3}\end{array}\right]$

$\operatorname{svd}(x)=\operatorname{norm}(x)=\sqrt{x_{1}^{2}+x_{2}^{2}+x_{3}^{2}}$

It is well known that the norm of a matrix is its largest $s v d$ value. However, for a one dimensional matrix it is a single value. The cost matrix is the arrangement of $s v d$ values of the difference vectors of the II of the two shapes, for each point of one shape against every point of the other shape. Here, the shape correspondence addresses three major issues in shape matching problems.

\subsubsection{Speed of Matching}

Pointwise correspondences should have the property that an occlusion in one part of a shape should not affect point-wise correspondences in other regions and the speed of matching should not degrade sharply. We have found that the FMA has the advantage over the more commonly-used Dijkstra's algorithm that it matches all regions independently rather than sequentially, as shown in Fig. 8. In this example, the occluded leg of the red shape is matched with the complete leg of the blue shape. Note that this has not affected the process of establishing correspondences in other regions. An example geodesic path obtained by the FMA overlaid over a feature map for this pair of shapes is given in Fig. 9. The geodesic path in the feature map is found using a gradient descent algorithm, as shown in Fig. 10. The twist in the path near the bottom indicates the high cost of matching, and reflects the apparent mismatch because of the occluded leg.

Figures 11 and 12 show examples of matching cost calculated for a family of quadrupeds and fish using the proposed method. Strong intra group similarity and intergroup dissimilarity is observed in the example.

\subsubsection{Initial Alignment}

A shape is a closed planar contour that can have any point designated as a starting point. However in shape correspondence problems, the starting points of the two shapes should match. Point-wise correspondence using II and FMA can be established even if two shapes are unaligned to a certain extent. However, if the shapes are substantially out of alignment, or one or both contain major occlusions, then a correct correspondence is difficult to achieve. This is precisely the problem we have addressed. The shape signature is divided
477

478

479

480

481

482

483

484 
(a)

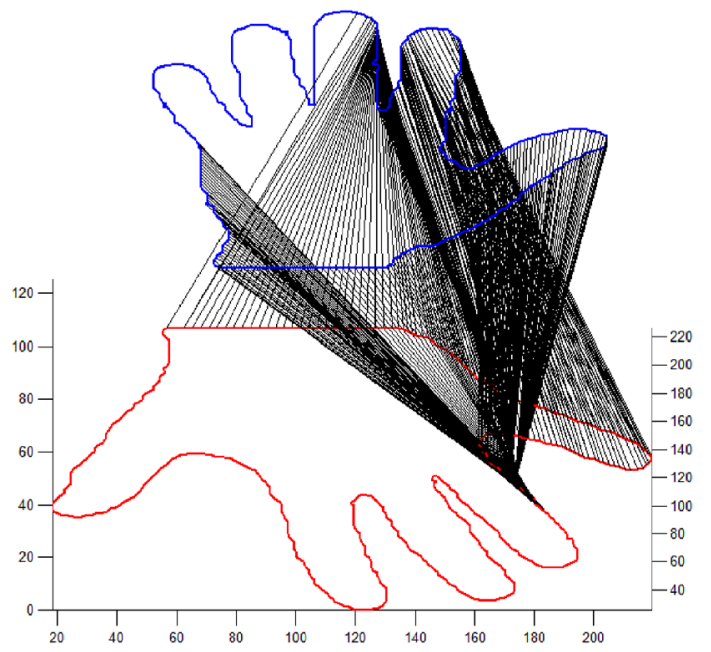

(c)
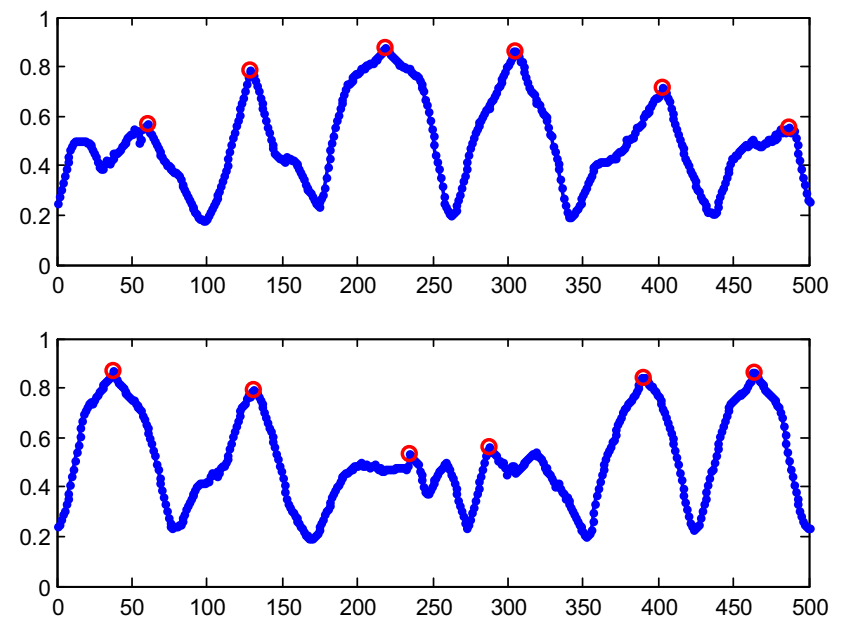

(e)

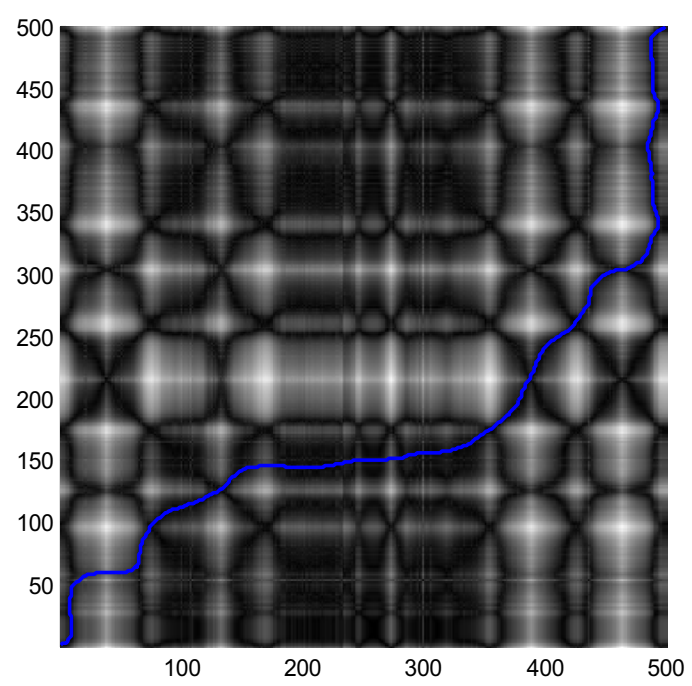

Fig. 13 Shape correspondence example of two hand shapes that are out of phase to each other. Shapes are wrongly corresponded without an initial alignment in the first column, while correctly corresponded in the second column after initial alignment. The second row compares (b)

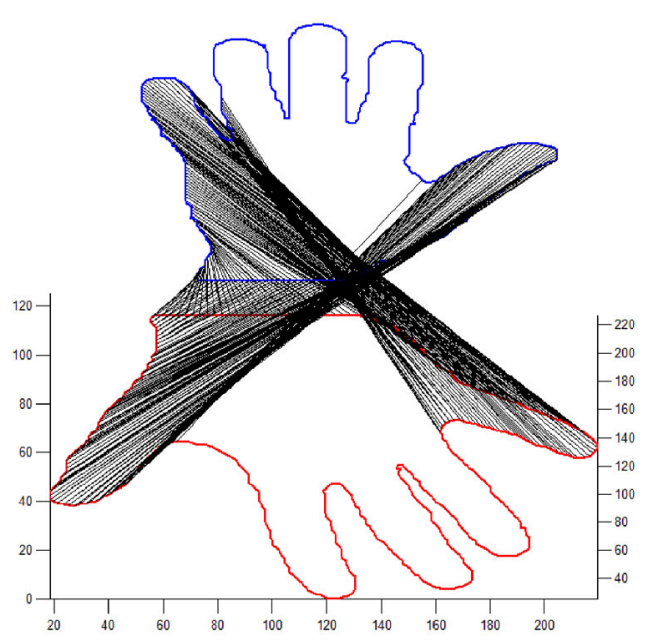

(d)
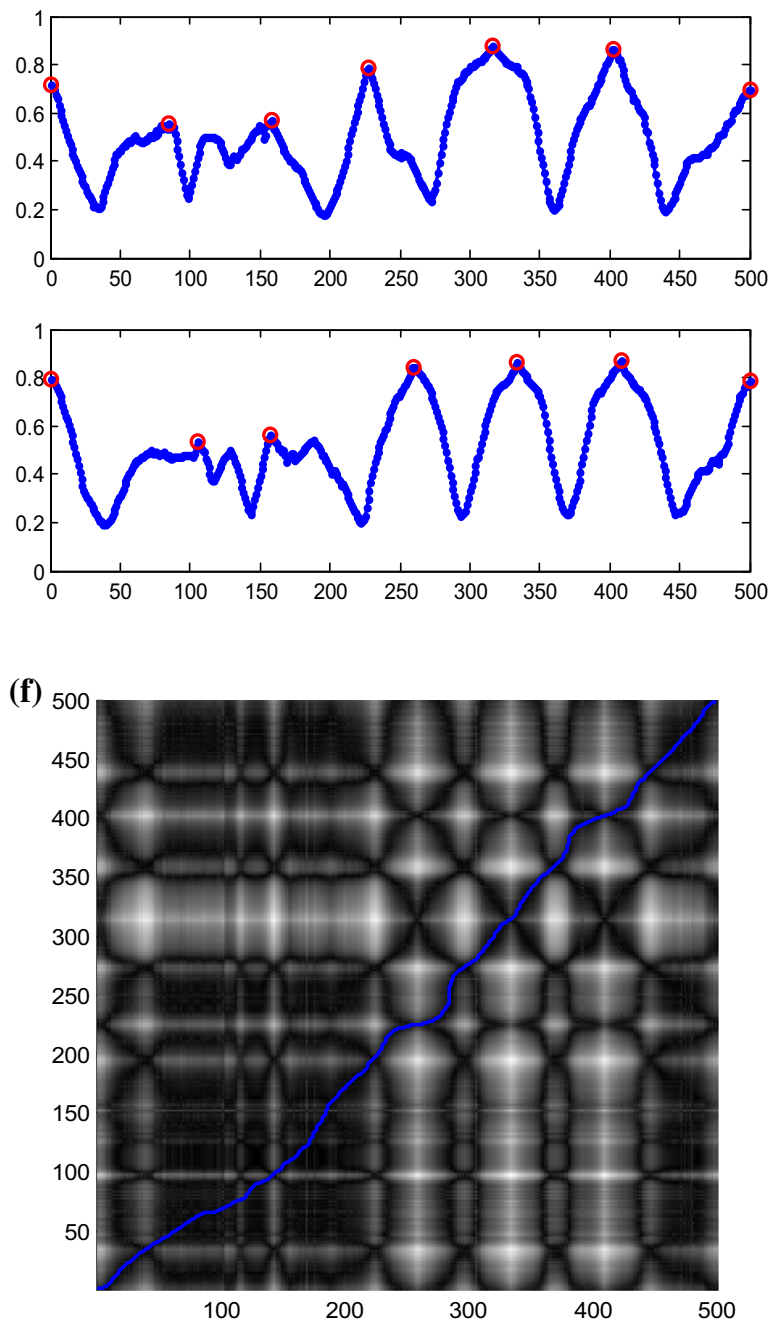

the shape signature at the coarsest scale for both shapes, whereas, the third row contains the shortest path superimposed on the feature space and compares the point-wise correspondence results, which is better for (f) 
into regions based on causal peaks of Integral invariant scale space and the starting points of the best matching regions in two shapes are designated as the points of initial alignment. Causality of a scale space means that finer scales of observation directly reflect what happens at the coarse scale. Integral invariants are applied at varying scales of observation (kernel size) to the shapes described for matching and the causal peaks describe its general overall structure. Figure 13 illustrates a shape correspondence of two hands, with and without initial alignment. From Fig. 13e, f, the geodesic path for initially aligned hands is quite short (straight) in diagonal, showing a closer match.

\subsubsection{Scale Selection}

A range of integral invariant scales is used to obtain the feature space given in Fig. 13 and consequently to maximize the difference between two shapes. Larger scales are locate and differentiate larger regions, whereas smaller scales are essential to distinguish fine details. Scale space reflects the saliency of regions that maintain causal peaks at all given scales. Shapes are divided into regions based upon peaks at the coarsest scale. Finding a suitable single coarse scale to correspond shapes with significantly large size ratios is tedious. Let $r_{\max }$ be the maximum scale indicator, then comparing shapes $\left(S_{1}, S_{2}\right)$ for correspondence where the area of shape to integral invariant ratio (SIR) is fixed, $r_{\max }$ is,

$$
\begin{aligned}
r_{\max } & =\left\lceil\operatorname { m e a n } \left( r_{S_{1 \max }}, r_{\left.\left.S_{2 \max }\right)\right\rceil,}\right.\right. \\
\text { where } r_{S_{\text {max }}} & =\sqrt{\frac{\text { Area of } S_{i}}{\operatorname{SIR} * \pi}}, i=[1,2]
\end{aligned}
$$

Though to a large extent, scale selection depends upon the size of the shape, we have observed experimentally that it also depends upon the variability in the shape boundary. To date, we have not found a generalized relationship between the two and consider it application dependent. This will form part of our future work.

\subsection{Computing Eccentricity Transformations}

The computation of Ecc was outlined in Sect. 3.2. First, geodesic distances for each point in a shape to every point on the boundary are calculated. As the goal is to find the farthest point; there is no reason to find distances between the points inside the boundary. Figure 14 shows the feature set, where geodesic distance of each point on the boundary to every point inside the shape is found. The result is a feature array for each point inside the shape with size $1 \times n$, where $n$ is the total number of samples on the boundary. The image size in this example is $200 \times 200$ and $n=500$. The Ecc shape signatures are computed from this feature space, depending upon the type of distance used. To date, we have used the
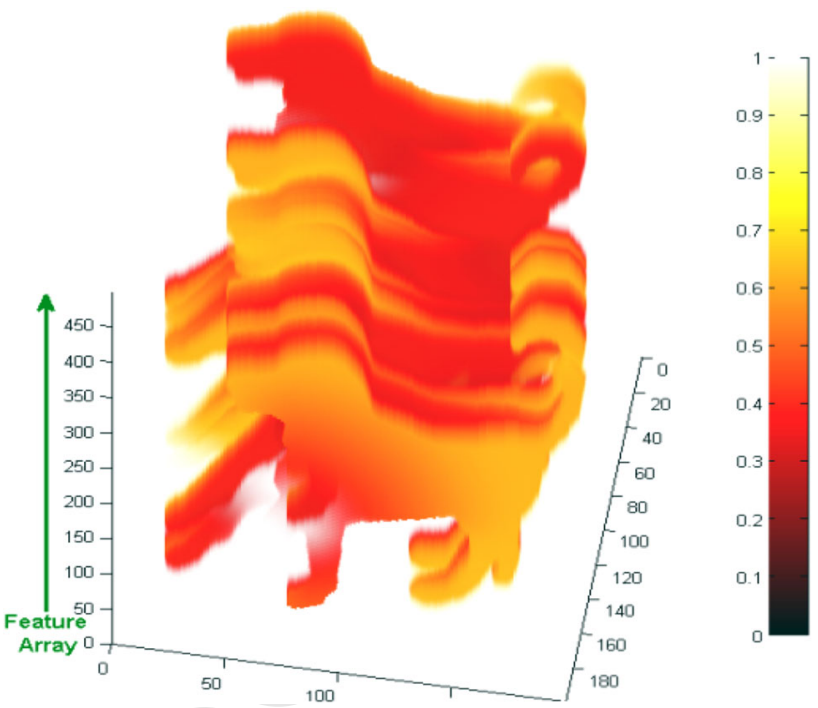

Fig. 14 Feature set of all the geodesic distances inside the shape to every point on the boundary. The farthest point, or the maximum distance, is calculated along the direction of the feature array. The first two dimensions are the size of the image, the third is the number of feature points on the shape, whereas each point inside each shape describes a distance, normalized and presented in the false colour map with a colour bar on the right hand side of the figure (Color figure online)
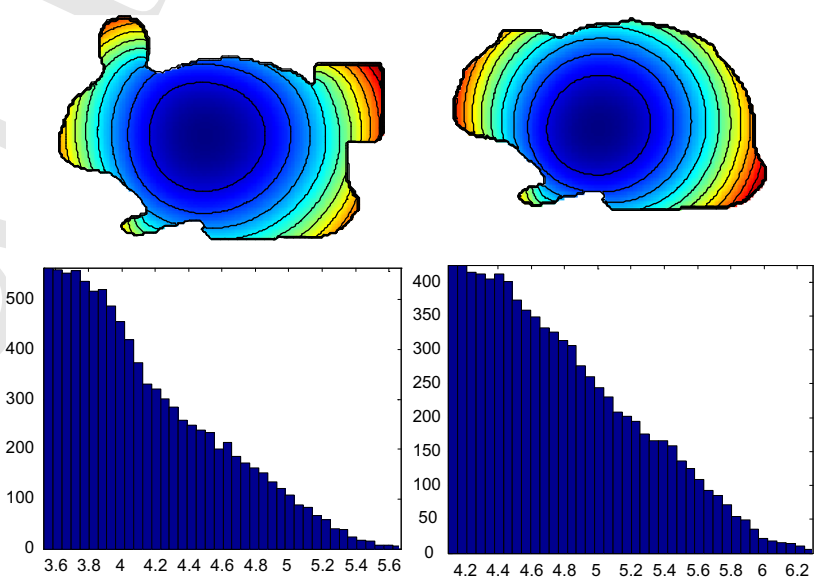

Fig. 15 Shape histogram signatures of two shapes from Kimia database. The Eccentricity transform fails to describe the difference in a meaningful way between the two shapes

mean distance across the $1 x n$ array, however other distances may also be used, as shown in Fig. 14.

\subsection{Combining Eccentricity Transform and Integral Invariant}

A fundamental limitation of II is that they relate only to the boundary and do not take into account the information from inside the shape. As a result, two similar geometric features on a shape boundary, but at very different locations, will produce the same shape signature. This may result in false 


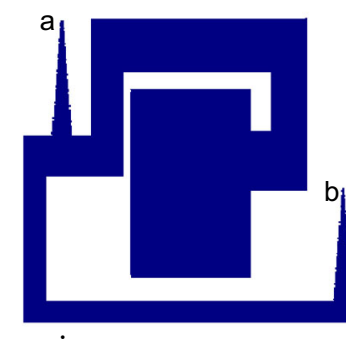

i

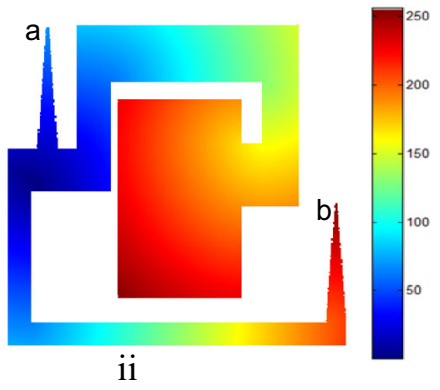

ii
Fig. 16 A shape (i), with Eccentricity (Ecc) transformation in (ii)

point-wise shape correspondences. On the other hand, the Ecc has been used successfully used for shape matching and retrieval; however, it cannot find differences between two shapes. For example, Fig. 15 shows two rabbit shapes with different occluded features; their Ecc signatures are shown underneath. It can be seen that the given shape signatures do not indicate shape differences.

We combine these two ideas, tuning Integral Invariant boundary signatures based on the eccentricity information about the locations within a shape. Figure 16 shows a shape with two pointed peaks, which have similar features, though in different locations. In the Eccentricity transformed version of shape; it is immediately apparent that the two peaks now contain different values in the false colour model. If we look at the Integral Invariant Signatures of the same shape before and after the application of Eccentricity transform, as shown in Fig. 17, the difference is evident and peaks are now distinguishable. The Integral Invariant shape signature, shown in blue, cannot differentiate between points ' $a$ ' and ' $b$ ', giving no clue about the location of these points inside a shape. The shape signature after applying the Eccentricity transform gives them a distinct meaning. Figure 18 shows two shapes, $S 1$ and $S 2$, with a pair of corresponding points, where we expect that $a 1$ corresponds to $a 2$ and $b 1$ corresponds to $b 2$. The method results in correct correspondences, rather than corresponding $a 1$ to $b 2$ and $a 2$ to $b 1$.

\subsection{Shape Matching Cost}

Once an Ecc image is acquired, a multi-scale approach is used for Integral Invariant (II) shape correspondence. The kernel size $r$ is varied to span a range of apertures. As a result, the Integral Invariant creates a scale space where for every two points $x \in S 1$ and $y \in S 2$, the sum of squared difference of the Integral Invariant is computed, and this forms a feature vector $V_{S}$. Since $V_{S}$ is a vector, it has a unique singular value and there is no need for computation of the largest singular value, which is considered to be the maximum distance between $x$ and $y$. In this way a similarity/distance matrix $D\left(S_{1}, S_{2}\right)$ is obtained, which contains the Integral Invariant difference for each point between two shapes. For shape correspondence, the FMA is applied to the similarity/distance matrix to find a distance map $\widehat{D}\left(S_{1}, S_{2}\right)$, and then the shortest geodesic path $G\left(S_{1}, S_{2}\right)$ from $D(0,0)$ to $D(n, n)$ is calculated using gradient descent, where $n$ is the parameterization of the curves $\partial S_{1}$ and $\partial S_{2}$. The matching cost between two shapes is given by,

$$
C\left(S_{1}, S_{2}\right) \stackrel{\text { def }}{=} \int_{D\left(S_{1}, S_{2}\right)} G\left(S_{1}(t), S_{2}(t)\right) d t
$$

II-on-Ecc takes into account the grey level values which is the resultant of the Ecc transformation on the shape, unlike the conventional II method which, as noted above, is blind to the shape contents.

\section{Results}

\subsection{Application to Synthetic Images}

We applied the algorithm to 36 shapes from the Kimia database-4 shapes each from the 9 shape categories. As the method is quite general, a broader evaluation may be carried out to evaluate precision of this method for a specific application. Though we have developed this algorithm to find corresponding regions of interest in pairs of mam-
Fig. 17 Normalized II signature (blue) of the original shape in Fig. 11. The II invariant signature of the Ecc version (II-on-Ecc-red). The portions $a$ and $b$, show how two similar features on a shape may be tuned to have distinct signatures based on their locality using proposed method (Color figure online)

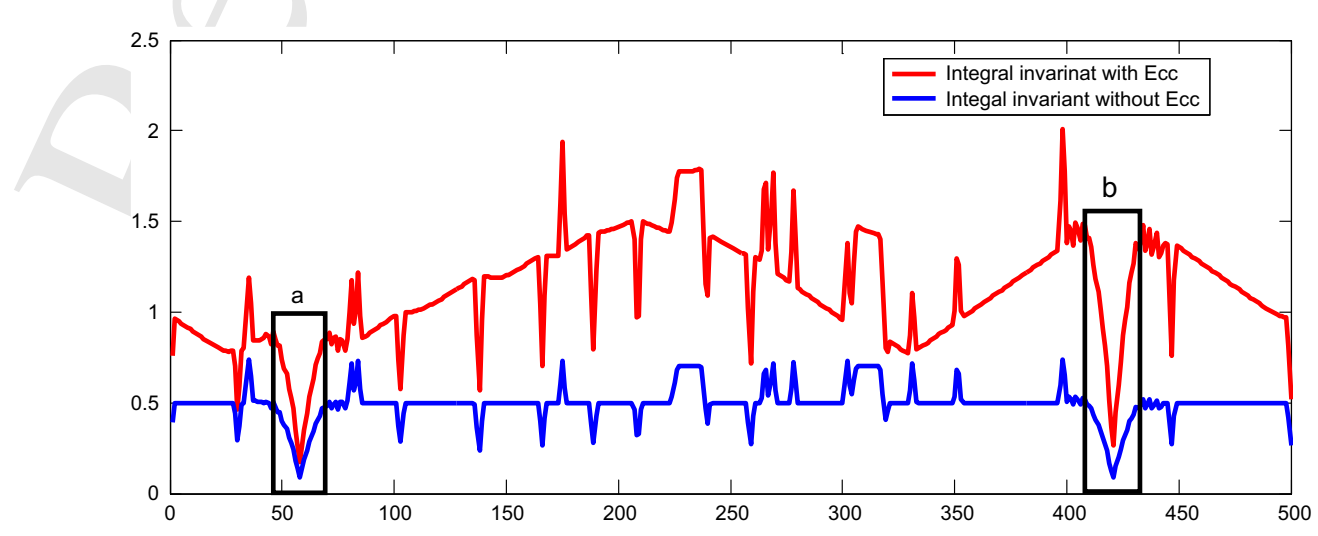


mograms taken at different times, we have used the Kimia database as it is currently considered to be a standard to assess shape matching algorithms. All pairs of shapes are compared and a matching cost is calculated for II, Ecc and II-on-Ecc matching. We find that II-on-Ecc gives the strongest intra group matching. Figure 19 summarizes the results. The dark blocks along the diagonal reflect the low cost of matching within a specific shape group, which means higher similarity. It can be seen that the application of the Eccentricity transform has enhanced the inter-group similarity. Figure 20 shows the shape retrieval results for this method. However, for our application, the method is focused on reducing correspondence errors, for which it shows considerable promise.

The charts in Fig. 20 show shape retrieval using: Integral Invariant; Eccentricity transforms; and finally Integral Invariant on Eccentricity transform shapes. The $\mathrm{X}$ and $\mathrm{Y}$ axis of the chart consists of shapes, which are indexed consecutively from 1 to 36 in 9 different shape groups from Kimia database. Each box represents a shape on the $\mathrm{x}$-axis, and its height (range) on the y axis represent top 4 matches among all 36 shapes. The red bar in each box shows the median shape value of retrieved matches. Categories of Rabbits, Aliens, Tools, Men and Kite have perfect group retrieval results for II-on-Ecc method. Overall, using the Eccentricity transform prior to Integral Invariant improves the results.
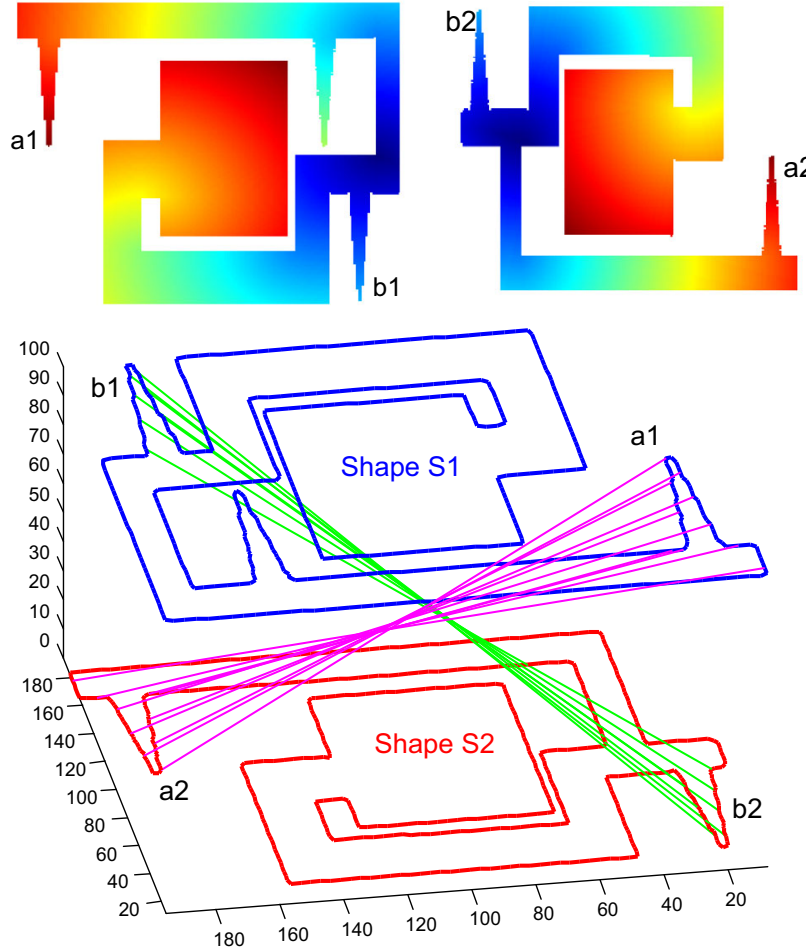

Fig. 18 Two Ecc transformed shapes (left) and its correct correspondence (right) using II and Fast Marching Algorithm. II without Ecc will incorrectly match points $b 1$ to $a 2$ and $a 1$ to $b 2$
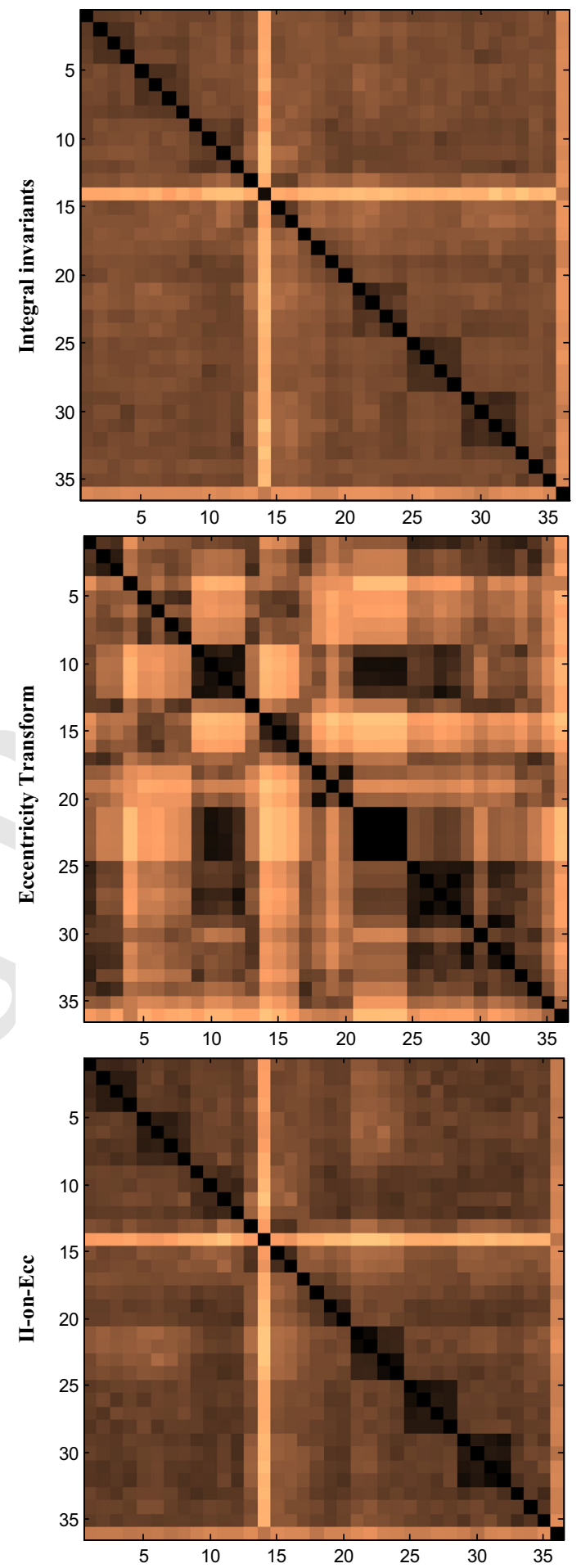

Fig. 19 Shape matching results of methods mentioned above. Dark pixels reflect a low matching cost and higher shape similarity, which is greater for II-on-Ecc. Refer to Fig. 20 for shape retrieval details

\subsection{Application to Mammograms}

Most Computer Aided Detection (CAD) systems use image processing algorithms to detect abnormalities in mammo- 
Fig. 20 Shape retrieval results using mentioned methods. a Integral Invariant shape retrieval with noise. b Eccentricity transform shape retrieval with noise. c Integral Invariant on Eccentricity transform shape retrieval with noise

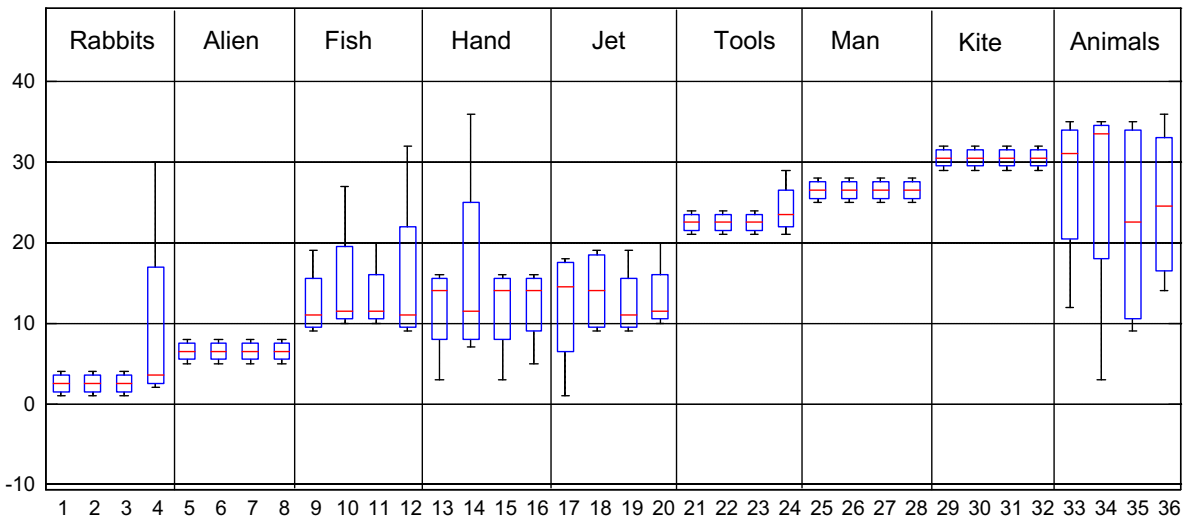

(a) Integral Invariant shape retrieval with noise

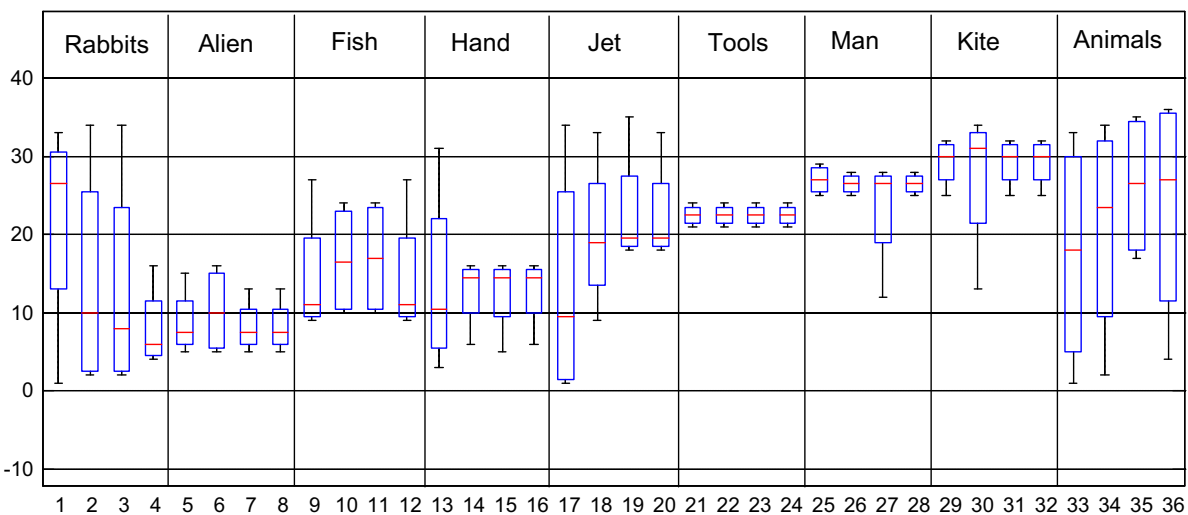

(b) Eccentricity transform shape retrieval with noise

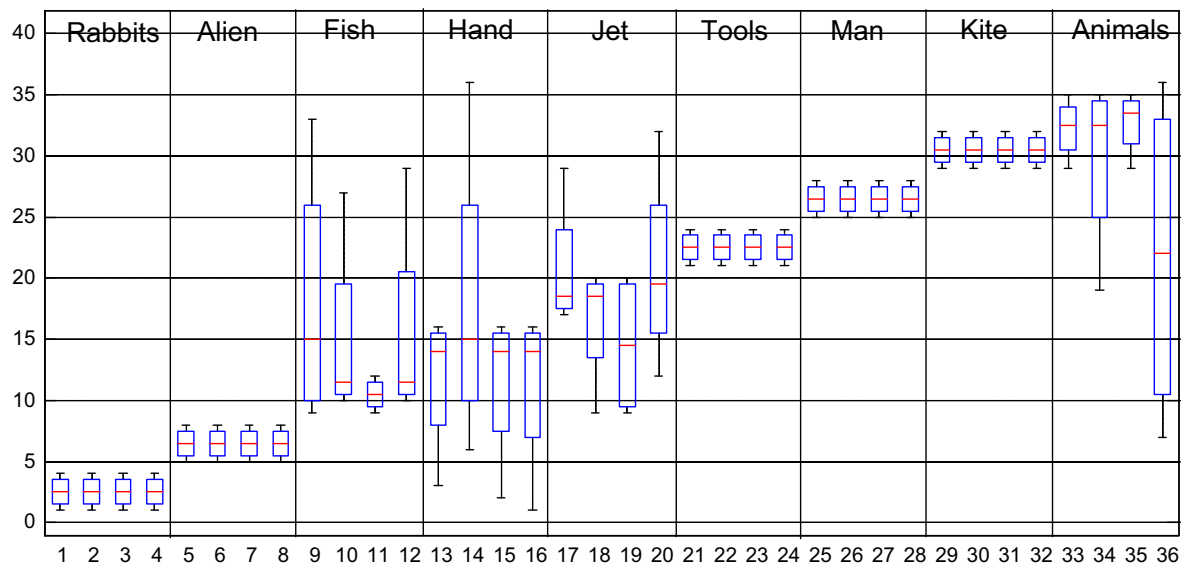

(c) Integral Invariant on Eccentricity transform shape retrieval with noise grams such as calcifications and masses, and to support the temporal study of mammograms and architectural distortions. The use of CAD technologies by radiologists and pathologists plays a key role in the early detection of breast cancer and helping to reduce the mortality rate among women (Sampat et al. 2005). The aim is to find changes in the region of interest, over time or in different views of the same mammogram. Because mammograms are such complex images and vary considerably over the population, it is common clinical practice in breast radiology to analyze two or more mammograms in order to detect anomalies. While comparing two mammograms of the same patient, the breasts may vary in size and in the way they are imaged; but the internal structure is quite similar and symmetric over large areas. We address the temporal study of mammograms by employing the proposed shape analysis technique for local region correspondence and matching in segmented mammograms. 

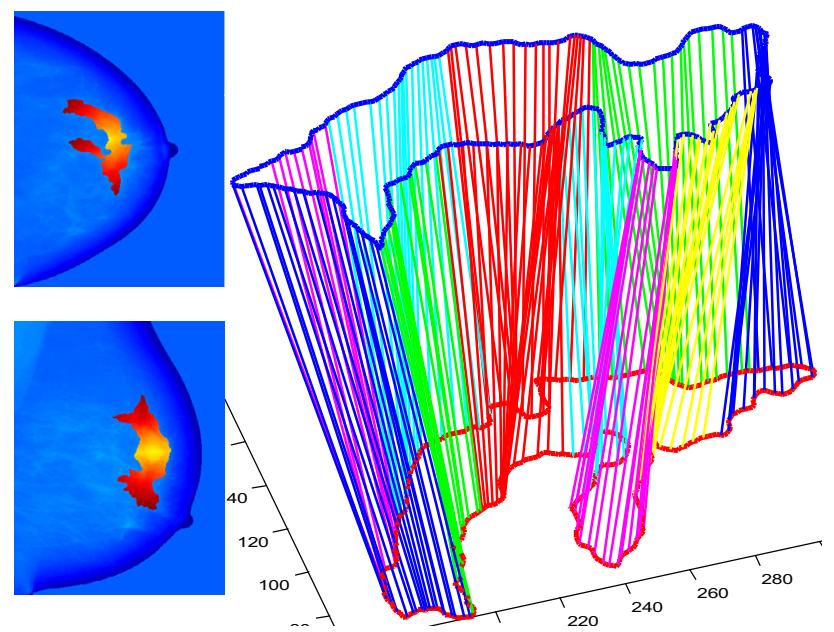

Fig. 21 Segmented pair of CC and MLO views of breast density maps, obtained by Volpara ${ }^{\circledR}$, matched and corresponded using our proposed method

We applied our Integral Invariant on Eccentricity transform algorithm to density mammograms. In this case, the aim is to find changes in regions of interest, over time or in different views of the same mammogram. Figure 21 shows a pair of Craniocaudal (CC) and Mediolateral oblique (MLO) breast density maps created by the commercial software Volpara $^{\circledR}$ (Highnam et al. 2010; Jeffreys et al. 2010). Both mammograms were automatically segmented using a hierarchical segmentation method (topographic approach Hong 2004; Hong and Brady 2003) based on iso-contours. As a result, a number of regions were identified and matched using the method described above. Two regions, suspected of being abnormalities are shown in Fig. 21, which show Eccentricity transformed shapes superimposed on density maps. The correspondence results are very encouraging. It may be noticed in Fig. 22 that II-on-Ecc performs better than II alone, where the geodesic path for II-on-Ecc shows a more regularised matching and consequently yield a lower matching cost for a closer match

The matrices shown in Fig. 22 are the feature maps, that are estimated using the method explained in Sect. 4.1. Both shapes, in this case, are parameterized to equal length and each point in these matrices is the greatest singular value difference for Integral Invariant values at each corresponding pair. Integral Invariant on Eccentricity transform gives a more regularized match, as said above, and the geodesic path overlaid on feature map is shorter and tended towards the diagonal.

A selection of examples of applying the method to mammograms is given in Fig. 23. A fuller assessment of the method applied to mammograms will be presented elsewhere. For the purposes of this paper it suffices to state that the method substantially reduces the number of false positive matches.
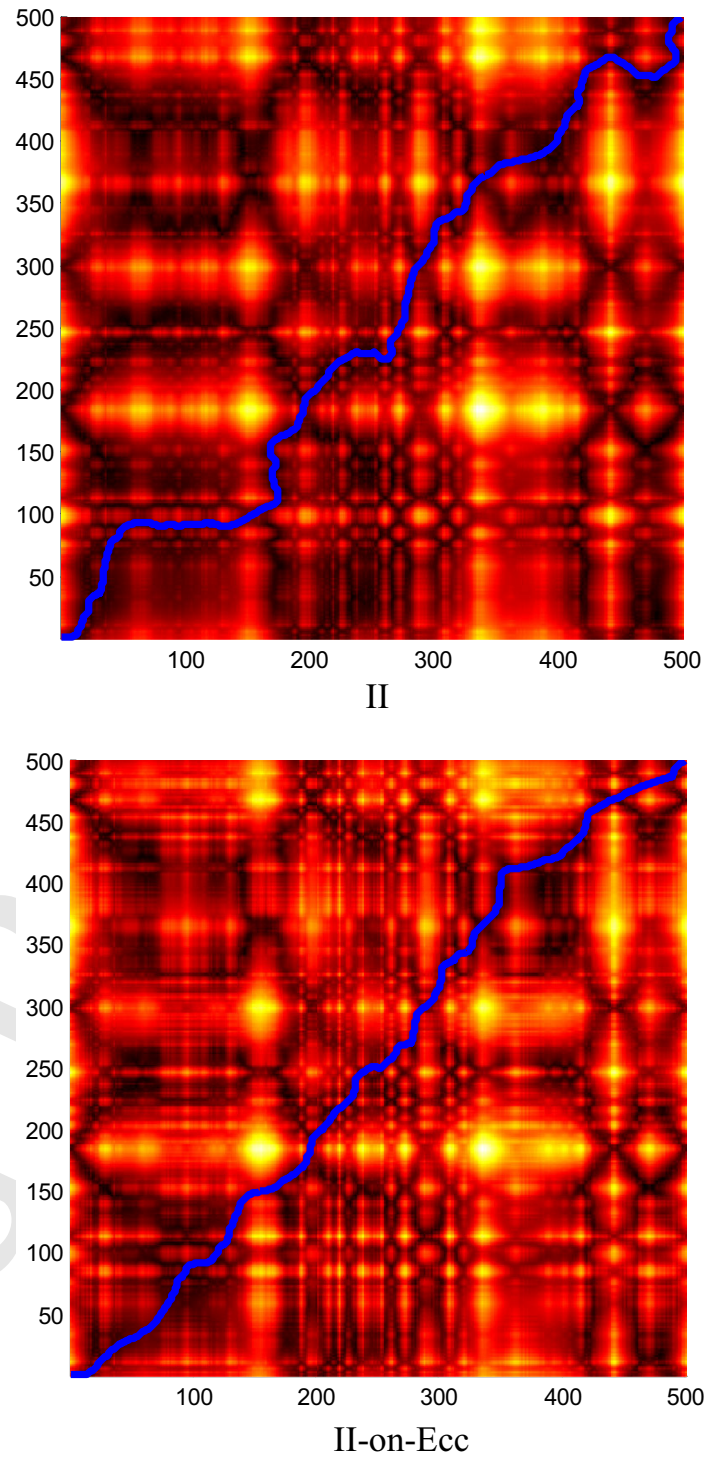

Fig. 22 Geodesic path drawn over similarity matrix, which shows point-wise correspondence between regions in Fig. 21. No results for Ecc are given here, as it cannot establish point-wise correspondence of shapes

Though we have applied this method to mammography for which we had resources readily available; it could be applied to a wide range of applications, potentially beyond medical imaging. One of the intrinsic limitations of temporal mammograms is that two breast images, taken at different times, while having the same clinical views, may, not least as a result of different breast compression, show different structures resulting in substantially different boundaries. Some of these changes are due to a number of biological factors; nevertheless, a change due to the positioning of the breast is an inevitable factor which could not be avoided. Difference in the imaging parameters also affect the sensitive of segmentation methods which are dependent upon the 


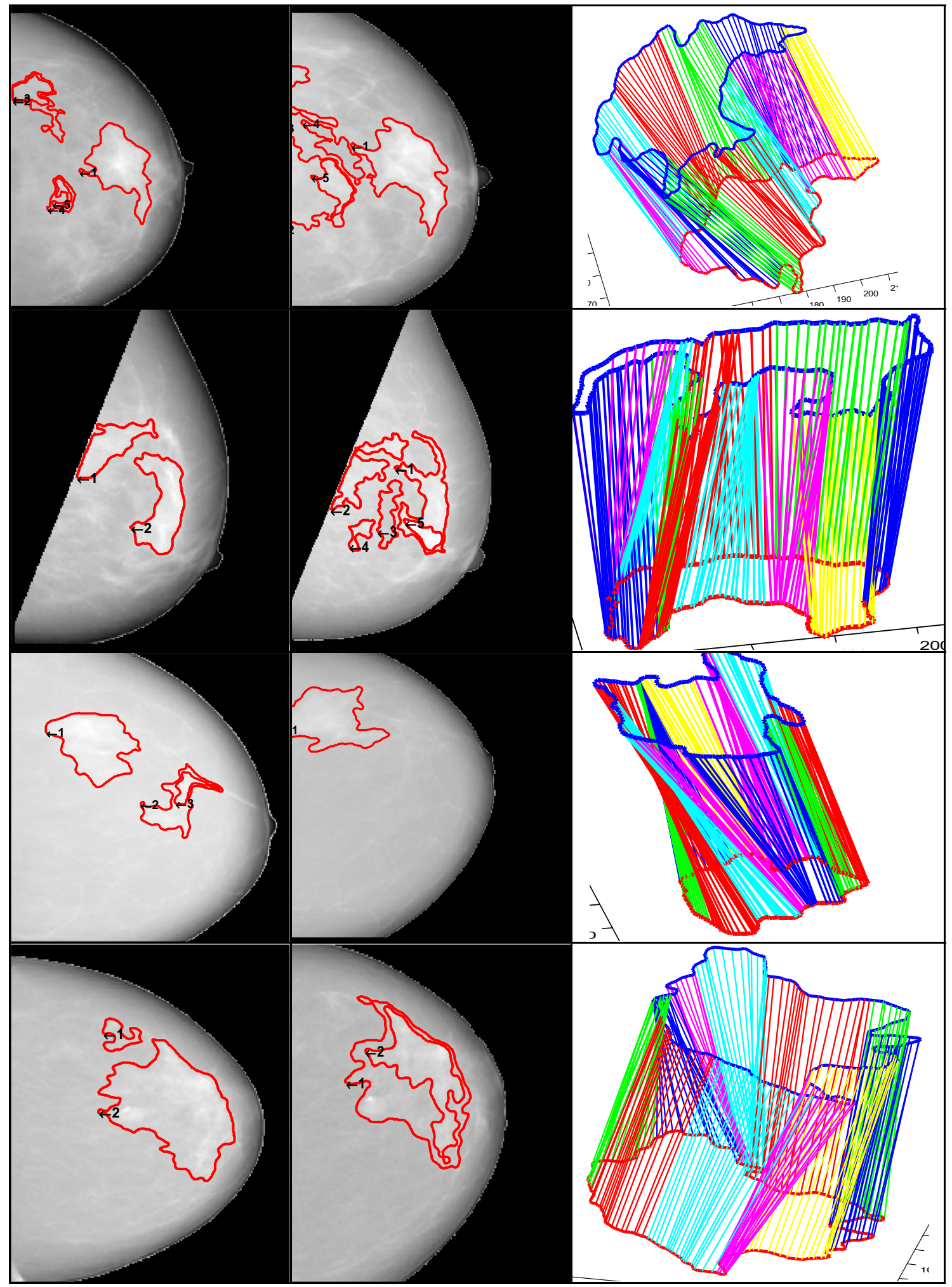

Fig. 23 A few more examples of segmented, temporal mammograms, where regions are matched and corresponded against each other using the proposed method 
gradient and intensities of mammographic regions, resulting in geometrically different shapes for matching. Having this in mind, this is a case study to compare temporal mammograms using the proposed method, which aims to emphasis the potential of the mentioned approach and does not argue the accuracy of the matched regions acquired after segmentation, which are subject to above limitations. A reliable segmentation method would considerably enhance the utility of the proposed method.

Referring to Fig. 22, it is noted that the geodesic path is regular and approaches the diagonal. This means that Ecc processing in a certain way compensates for the deformation and generates a kind of inverse transformation of these deformations. However, further analysis using deformation model remains as future work.

\section{Discussion}

In this paper, we have combined structural and boundary information in a shape matching application, and applied the method to establish regional correspondences in temporal pairs of mammograms. Both the Integral Invariant and Eccentricity transforms are invariant to isometric deformations, such as bending and articulations. However, II are a contour-based local descriptor, which relates to the boundary of the shape and do not take into account the inside of a shape. On the other hand, Ecc is a global region based descriptor that maps the structural anatomy of a shape, however, does not explicitly contain the boundary information, including curvature. We describe a method that combines both techniques by tuning the Integral Invariant boundary signature based on the eccentric information about regions within the shape.

The experimental results presented here show qualitative improvement compared to Integral Invariant results; however, the main aim of this method is to reduce correspondence errors while matching two shapes. Shape matching algorithms usually become stuck in local minima while establishing regional correspondences. This method first stretches regional differences within each shape, thus emphasizing dissimilarities before comparing them, which reduces the probability of false correspondences. This feature is the fundamental strength of our approach.

We applied the method to shapes from the Kimia database and compared the results to those obtained by Integral Invariant and Eccentricity transforms when applied separately. There is an overall improvement in results for both inter and intra group shape matching. The Fast Marching Algorithm was applied to establish a point-wise correspondence between shapes and to calculate a matching cost. The results are encouraging and indicate scope for further improvement.
One of the limitations of our method is that there is a trade-off between the descriptiveness and invariance of any shape descriptor. However, since the Eccentricity transform is invariant to Euclidean transformations, it is anticipated that the descriptiveness it adds to the Integral Invariant signature would not be significant.

\section{References}

Alferez, R., \& Wang, Y.-F. (1999). Geometric and illumination invariants for object recognition. IEEE Transactions on Pattern Analysis and Machine Intelligence, 21, 505-536.

Amanatiadis, A., Kaburlasos, V. G., Gasteratos, A., \& Papadakis, S. E. (2011). Evaluation of shape descriptors for shape-based image retrieval. Image Process IET, 5, 493-499.

Arrebola, F., \& Sandoval, F. (2005). Corner detection and curve segmentation by multiresolution chain-code linking. Pattern Recognition, 38, 1596-1614.

Arun, K. S., \& Sarath, K. S. (2011). Evaluation of SUSAN filter for the identification of micro calcification. International Journal of Computational and Applied, 15, 41-44.

Bauer, M., Fidler, T., \& Grasmair, M. (2011). Local uniqueness of the circular integral invariant. arXiv Prepr. arXiv:1107.4257.

Belongie, S., Malik, J., \& Puzicha, J. (2002). Shape matching and object recognition using shape contexts. IEEE Transactions on Pattern Analysis and Machine Intelligence, 24, 509-522.

Belongie, S., Malik, J., \& Puzicha, J. (2001). Matching shapes. In: Proceedings of the 8th IEEE International Conference on Computer Vision ICCV. ICCV 2001 (pp. 454-461).

Bengtsson, A., \& Eklundh, J.-O. (1991). Shape representation by multiscale contour approximation. IEEE Transactions on Pattern Analysis and Machine Intelligence, 13, 85-93.

Bertsekas, D. P. (1995). Dynamic programming and optimal control. Belmont, MA: Athena Scientific.

Und Bildverarbeitung, A.M. \& Ion, D.-IA. (2009). The Eccentricity Transform of n-Dimensional Shapes with and without Boundary.

Boué, M., \& Dupuis, P. (1999). Markov chain approximations for deterministic control problems with affine dynamics and quadratic cost in the control. SIAM Journal on Numerical Analysis, 36, 667-695.

Brandt, R. D., \& Lin, F. (1996). Representations that uniquely characterize images modulo translation, rotation, and scaling. Pattern Recognition Letters, 17, 1001-1015.

Bronstein, A. M., Bronstein, M. M., Bruckstein, A. M., \& Kimmel, R. (2008). Analysis of two-dimensional non-rigid shapes. International Journal of Computer Vision, 78, 67-88.

Bronstein, A. M., Bronstein, M. M., \& Kimmel, R. (2006). Generalized multidimensional scaling: A framework for isometry-invariant partial surface matching. Proceedings of the National Academy of Sciences United States of America, 103, 1168-1172.

Bruckstein, A. M., Rivlin, E., \& Weiss, I. (1997). Scale space semi-local invariants. Image and Vision Computing, 15, 335-344.

Calabi, E., Olver, P. J., Shakiban, C., et al. (1998). Differential and numerically invariant signature curves applied to object recognition. International Journal of Computer Vision, 26, 107-135.

Cao, W., Hu, P., Liu, Y., et al. (2011). Gaussian-curvature-derived invariants for isometry. Science China Information Sciences, 56(9), 112.

Chen, Y. W., \& Xu, C. L. (2009). Rolling penetrate descriptor for shapebased image retrieval and object recognition. Pattern Recognition Letters, 30, 799-804.

Chetverikov, D., \& Khenokh, Y. (1999). Matching for shape defect detection. Computer Analysis Images Patterns. pp. 367-374. 
Cohen, F. S., \& Wang, J.-Y. (1994). Part I: Modeling image curves using invariant 3-D object curve models-a path to 3-D recognition and shape estimation from image contours. IEEE Transactions on Pattern Analysis and Machine Intelligence, 16, 1-12.

Cohen, L. D., \& Kimmel, R. (1997). Global minimum for active contour models: A minimal path approach. International Journal of Computer Vision, 24, 57-78.

Cohignac, T., Lopez, C., \& Morel, J. M. (1994). Integral and local affine invariant parameter and application to shape recognition. Pattern Recognition, 1994. Vol. 1-Conference A: Comput. Vis. \& Image Process. In: Proceedings of 12th IAPR International Conference (pp. 164-168).

Cole, J. B., Murase, H., \& Naito, S. (1991). A Lie group theoretic approach to the invariance problem in feature extraction and object recognition. Pattern Recognition Letters, 12, 519-523.

Davidovic, T., Ramljak, D., Selmic, M., \& Teodorovic, D. (2010). Parallel bee colony optimization for scheduling independent tasks on identical machines. Proceedings of International Symposium on Operational Research (pp. 389-392).

Davies, E. R. (2004). Machine vision: Theory, algorithms, practicalities. Boston: Elsevier.

Davis, L. S. (1977). Understanding shape: Angles and sides. IEEE Transactions on Computers, 100, 236-242.

Dijkstra, E. W. (1968). Co-operating sequential processes. New York: F. Program. Lang. Acad. Press.

Dijkstra, E. W. (1959). A note on two problems in connexion with graphs. Numerical Mathematics, 1, 269-271.

Dijkstra, E. W. (1976). A discipline of programming. Englewood Cliffs, NJ: Prentice-Hall.

Duchenne, O., Bach, F., Kweon, I.-S., \& Ponce, J. (2011). A tensorbased algorithm for high-order graph matching. IEEE Transactions on Pattern Analysis and Machine Intelligence, 33, 2383-2395.

Duci, A., Yezzi, A. J., Mitter, S. K., \& Soatto, S. (2003). Shape representation via harmonic embedding. Proceedings 9th IEEE International Conference on Computer Vision, 2003 (pp. 656-662).

Elad, A., \& Kimmel, R. (2003). On bending invariant signatures for surfaces. IEEE Transactions on Pattern Analysis and Machine Intelligence, 25, 1285-1295.

Fidler, T., Grasmair, M., Pottmann, H., \& Scherzer, O. (2007). Inverse problems of integral invariants and shape signatures.

Forsyth, D., Mundy, J. L., Zisserman, A., et al. (1991). Invariant descriptors for $3 \mathrm{~d}$ object recognition and pose. IEEE Transactions on Pattern Analysis and Machine Intelligence, 13, 971-991.

Forsyth, D., Mundy, J. L., Zisserman, A., \& Brown, C. M. (1990). Projectively invariant representations using implicit algebraic curves. Berlin: Springer.

Frenkel, M., \& Basri, R. (2003). Curve matching using the fast marching method. Energy Minimization Methods in Computer Vision and Pattern Recognition, 2683, 35-51.

Gdalyahu, Y., \& Weinshall, D. (1999). Flexible syntactic matching of curves and its application to automatic hierarchical classification of silhouettes. IEEE Transactions on Pattern Analysis and Machine Intelligence, $21,1312-1328$.

Van Gool, L., Moons, T., \& Ungureanu, D. (1996). Affine/photometric invariants for planar intensity patterns. Computer VisionECCV'96. Berlin: Springer.

Gorelick, L., Galun, M., Sharon, E., et al. (2006). Shape representation and classification using the poisson equation. IEEE Transactions on Pattern Analysis and Machine Intelligence, 28, 1991-2005.

Gu, Y.-H., \& Tjahjadi, T. (2000). Coarse-to-fine planar object identification using invariant curve features and B-spline modeling. Pattern Recognition, 33, 1411-1422.

Hadley, G. (1964). Nonlinear and Dynamic Programming. Berlin: Addison-Wesley.

Hamza, A. B., \& Krim, H. (2006). Geodesic matching of triangulated surfaces. IEEE Transactions on Image Processing, 15, 2249-2258.
Hann, C., \& Hickman, M. S. (2002). Projective curvature and integral invariants. Acta Applied Mathematics, 74, 177-193.

Helgason, S. (1984). Groups \& geometric analysis: Radon transforms, invariant differential operators and spherical functions. Burlington, ON: Elsevier.

Helmsen, J., Puckett, E., Colella, P., \& Dorr, M. (1996). Two new methods for simulating photolithography development in 3D. In: Proceedings of SPIE (pp. 253-261).

Highnam, R., Brady, M., Yaffe, M. J., et al. (2010). Robust breast composition measurement-volparaTM. Digital mammography (pp. 342-349). Berlin: Springer.

Hong, B. W. (2004). Image segmentation, shape, and registration: Application to mammography. Oxford: University of Oxford.

Hong, B-W., \& Brady, M. (2003). Segmentation of mammograms in topographic approach. In VIE 2003. International Conference on Visual Information Engineering (pp. 157-160).

Huang, C.-L., \& Huang, D.-H. (1998). A content-based image retrieval system. Image and Vision Computing, 16, 149-163.

Huang, Q. X., Flöry, S., Gelfand, N., et al. (2006). Reassembling fractured objects by geometric matching. ACM Transactions on Graphics, 25(3), 569-578.

Huang, Z., \& Cohen, F. S. (1996). Affine-invariant B-spline moments for curve matching. IEEE Transactions on Image Processing, 5, $1473-1480$.

Ion, A., Artner, N. M., Peyré, G., et al. (2011). Matching 2D and $3 \mathrm{D}$ articulated shapes using the eccentricity transform. Computer Vision and Image Understanding, 115, 817-834.

Ion, A., Artner, N. M., \& Peyré, G., et al. (2008). 3D shape matching by geodesic eccentricity. In: IEEE Computer Society Conference on Computer Vision and Pattern Recognition Work 2008. CVPRW'08 (pp. 1-8).

Ion, A., Peyré, G., \& Haxhimusa, Y., et al. (2007). Shape matching using the geodesic eccentricity transform-a study. In: Proceedings of 31 st Annual Workshop Austrian Association Pattern (pp. 97-104).

Janan, F., \& Brady, M. (2012). Region matching in the temporal study of mammograms using integral invariant scale-space. Breast imaging (pp. 173-180). Berlin: Springer.

Jeffreys, M., Harvey, J., \& Highnam, R. (2010). Comparing a new volumetric breast density method (VolparaTM) to cumulus. Digital mammography. Berlin: Springer.

Van Kaick, O., Hamarneh, G., Zhang, H., \& Wighton, P. (2007). Contour correspondence via ant colony optimization. In:Proceedings of the 15th Pacific Conference on Computer Graphics and Applications (pp. 271-280).

Van Kaick, O., Zhang, H., Hamarneh, G., \& Cohen-Or, D. (2011). A survey on shape correspondence. Computer Graphics Forum, 30, $1681-1707$.

Kanatani, K. (1990). Group-theoretical methods in image understanding. Berlin: Springer.

Kendall, D. G. (1984). Shape manifolds, procrustean metrics, and complex projective spaces. Bulletin of the London Mathematical Society, 16, 81-121.

Kimmel, R. (2004). Fast marching methods. Numerical geometry of images. New York: Springer.

Kimmel, R., \& Sethian, J. A. (1996). Fast marching methods for robotic navigation with constraints. Berkeley, CA: Center for Pure and Applied Mathematics Report, University of California.

Kimmel, R., \& Sethian, J. A. (2001). Optimal algorithm for shape from shading and path planning. Journal of Mathematical Imaging and Vision, 14, 237-244.

Kliot, M., \& Rivlin, E. (1998). Invariant-based shape retrieval in pictorial databases. Computer vision-ECCV'98. Berlin: Springer.

Lasenby, J., Bayro-Corrochano, E., Lasenby, A. N., \& Sommer, G. (1996). A new framework for the formation of invariants and multiple-view constraints in computer vision. In: Proceedings of 
International Conference on Image Processing 1996 (pp. 313316).

Latecki, L. J., Lakamper, R., \& Eckhardt, T. (2000). Shape descriptors for non-rigid shapes with a single closed contour. In: Proceedings of IEEE Conference of Computer Vision Pattern Recognition 2000 (pp. 424-429).

Lenz, R. (1990). Group theoretical methods in image processing. New York: Springer.

Leordeanu, M., \& Hebert, M. (2005). A spectral technique for correspondence problems using pairwise constraints. In: Proceedings of 10th IEEE Intrernational Conference on Computer Vision, 2005. ICCV 2005 (pp. 1482-1489).

Ling, H., \& Jacobs, D. W. (2007). Shape classification using the innerdistance. IEEE Transactions on Pattern Analysis and Machine Intelligence, 29, 286-299.

Li, S. Z. (1992). Matching: Invariant to translations, rotations and scale changes. Pattern Recognition, 25, 583-594.

Li, S. Z. (1999). Shape matching basedon invariants. In O. M. Omidvar (Ed.), Progress in neural networks: Shape recognition (Vol. 6, pp. 203-228). Intellect.

Maciel, J., \& Costeira, J. P. (2003). A global solution to sparse correspondence problems. IEEE Transactions on Pattern Analysis and Machine Intelligence, 25, 187-199.

Manay, S., Cremers, D., Hong, B.-W., et al. (2006). Integral invariants for shape matching. IEEE Transactions on Pattern Analysis and Machine Intelligence, 28, 1602-1618.

Manay, S., Hong, B.-W., Yezzi, A. J., \& Soatto, S. (2004). Integral invariant signatures. Berlin: Springer.

Mansoory, M. S., Ashtiyani, M., \& Sarabadani, H. (2011). Automatic Crack Detection in Eggshell Based on SUSAN Edge Detector Using Fuzzy Thresholding. Modern Applied Science 5

Mardia, K. V., \& Dryden, I. L. (1989). Shape distributions for landmark data. Advances in Applied Probability, 21, 742-755.

Mateus, D., Horaud, R., \& Knossow, D., et al. (2008). Articulated shape matching using Laplacian eigenfunctions and unsupervised point registration. In: IEEE Conference on Computer Vision Pattern Recognition, 2008. CVPR 2008 (pp. 1-8).

Mikolajczyk, K., \& Schmid, C. (2005). A performance evaluation of local descriptors. IEEE Transactions on Pattern Analysis and Machine Intelligence, 27, 1615-1630.

Mokhtarian, F., Abbasi, S., \& Kittler, J. (1997). Efficient and robust retrieval by shape content through curvature scale space. Software Engineering and Knowledge Engineering, 8, 51-58.

Mokhtarian, F., \& Mackworth, A. (1986). Scale-based description and recognition of planar curves and two-dimensional shapes. IEEE Transactions on Pattern Analysis and Machine Intelligence, 8(1), 34-43.

Mokhtarian, F., \& Mackworth, A. K. (1992). A theory of multiscale, curvature-based shape representation for planar curves. IEEE Transactions on Pattern Analysis and Machine Intelligence, 14, 789-805.

Mumford, D. (1991). Mathematical theories of shape: Do they model perception? San Diego',91. San Diego, CA: Academic Press.

Mumford, D., Latto, A., \& Shah, J. (1984) The representation of shape. In: Proceedings of IEEE Workshop Computer Vision (pp. 183191).

Nasreddine, K., Benzinou, A., \& Fablet, R. (2009). Shape geodesics for boundary-based object recognition and retrieval. Image Process, pp. 405-408.

Nielsen, L., \& Sparr, G. (1991). Projective area-invariants as an extension of the cross-ratio. CVGIP: Image Understanding, 54, 145159.

Olver, P. J. (1995). Equivalence, invariants and symmetry. Cambridge: Cambridge University Press.

Osada, R., Funkhouser, T., Chazelle, B., \& Dobkin, D. (2002). Shape distributions. ACM Transactions on Graphics, 21, 807-832.
Ozcan, E., \& Mohan, C. K. (1997). Partial shape matching using genetic algorithms. Pattern Recognition Letters, 18, 987-992.

Petrakis, E. G. M., Diplaros, A., \& Milios, E. (2002). Matching and retrieval of distorted and occluded shapes using dynamic programming. IEEE Transactions on Pattern Analysis and Machine Intelligence, 24, 1501-1516.

Peyré, G. (2011). The numerical tours of signal processing part 2: Multiscale processings. Computing in Science \& Engineering, 13(5), 68-71.

Peyré, G., Péchaud, M., Keriven, R., \& Cohen, L. D. (2010). Geodesic methods in computer vision and graphics. Foundations and Trends in Computer Graphics and Vision, 5, 197-397.

Pottmann, H., Wallner, J., Huang, Q.-X., \& Yang, Y.-L. (2009). Integral invariants for robust geometry processing. Computer Aided Geometric Design, 26, 37-60.

Qu, Z.-G., Wang, P., Gao, Y.-H., \& Wang, P. (2011). Randomized SUSAN edge detector. Optical Engineering, 50, 110502-110502.

Rafajlowicz, E. (2007). SUSAN edge detector reinterpreted, simplified and modified. Multidimensional, pp. 69-74.

Reiss, T. H. (1993). Recognizing planar objects using invariant image features. New York: Springer.

Reuter, M., Wolter, F-E., \& Peinecke, N. (2005). Laplace-spectra as fingerprints for shape matching. In: Proceedings of 2005 ACM Symposium on Solid Physical Modelling (pp. 101-106).

Rezai-Rad, G., \& Aghababaie, M. (2006). Comparison of SUSAN and sobel edge detection in MRI images for feature extraction. In: Information and Communication Technologies 2006. ICTTA'06. 2nd (pp. 1103-1107).

Rosin, P. L. (2011). Shape description by bending invariant moments. Computer Analysis of Images and Patterns (pp. 253-260). Berlin: Springer.

Rothwell, C. A., Zisserman, A., Forsyth, D. A., \& Mundy, J. L. (1995), Planar object recognition using projective shape representation. International Journal of Computer Vision, 16, 57-99.

Rothwell, C. A., Zisserman, A., Forsyth, D. A., \& Mundy, J. L. (1992) Canonical frames for planar object recognition. Computer visionECCV'92 (pp. 757-772). Berlin: Springer.

Ruggeri, M. R., Patanè, G., Spagnuolo, M., \& Saupe, D. (2010) Spectral-driven isometry-invariant matching of 3D shapes. International Journal of Computer Vision, 89, 248-265.

Rusinol, M., Dosch, P., \& Lladós, J. (2007). Boundary shape recognition using accumulated length and angle information. Pattern Recognition and Image Analysis. Berlin: Springer.

Sampat, M. P., Markey, M. K., \& Bovik, A. C. (2005). Computer-aided detection and diagnosis in mammography. Handbook of Image and Video Processing, 2, 1195-1217.

Sato, J., \& Cipolla, R. (1997). Affine integral invariants for extracting symmetry axes. Image and Vision Computing, 15, 627-635.

Sato, J., \& Cipolla, R. (1996). Affine integral invariants and matching of curves. In: Proceedings of 13th International Conference on Pattern Recognition, 1996 (pp. 915-919).

Sebastian, T. B., Klein, P. N., \& Kimia, B. B. (2003). On aligning curves. IEEE Transactions on Pattern Analysis and Machine Intelligence, $25,116-125$.

Sebastian, T. B., Klein, P. N., \& Kimia, B. B. (2001). Alignment-based recognition of shape outlines. Visual Form 2001. Berlin: Springer.

Sethian, J. A. (1999). Level set methods and fast marching methods: Evolving interfaces in computational geometry, fluid mechanics, computer vision, and materials science. Cambridge: Cambridge University Press.

Sharma, A., \& Horaud, R. (2010). Shape matching based on diffusion embedding and on mutual isometric consistency. In: Computer Vision and Pattern Recognition Workshops (pp. 29-36).

Sharon, E., \& Mumford, D. (2006). 2d-shape analysis using conformal mapping. International Journal of Computer Vision, 70, 55-75. 
Shashua, A., \& Navab, N. (1996). Relative affine structure: Canonical model for 3D from 2D geometry and applications. IEEE Transactions on Pattern Analysis and Machine Intelligence, 18, 873-883.

Shi, J., Chen, F., Lu, J., \& Chen, G. (2013). An evolutionary image matching approach. Applied Soft Computing, 13, 3060-3065.

Siddiqi, K., Shokoufandeh, A., Dickinson, S. J., \& Zucker, S. W. (1999). Shock graphs and shape matching. International Journal of Computer Vision, 35, 13-32.

Si-ming, H., Bing-han, L., \& Wei-zhi, W. (2011). Moving shadow detection based on Susan algorithm. In: IEEE International Conference on Computer Science and Automation Engineering (pp. 16-20).

Smith, S. M., \& Brady, J. M. (1997). SUSAN: A new approach to low level image processing. International Journal of Computer Vision, $23,45-78$.

Sniedovich, M. (2010). Dynamic programming: Foundations and principles. Boca Raton, FL: CRC Press.

Sonka, M., Hlavac, V., \& Boyle, R. (1999). Image processing, analysis, and machine vision. London: Chapman and Hall Publishers.

Squire, D. M., \& Caelli, T. M. (2000). Invariance signatures: Characterizing contours by their departures from invariance. Computer Vision and Image Understanding, 77, 284-316.

Sundar, H., Silver, D., Gagvani, N., \& Dickinson, S. (2003). Skeleton based shape matching and retrieval. Shape Modeling International, 2003, 130-139.

Taubin, G., \& Cooper, D. B. (1991). Object recognition based on moment (or algebraic) invariants. IBM TJ Watson Research Center.

Teodorovic, D., Davidovic, T., \& Selmic, M. (2011). Bee colony optimization: The applications survey. ACM Transactions on Computational Logic, 1529, 3785.

Thomas, T. Y. (1934). The differential invariants of generalized spaces. Cambridge: Cambridge University Press.

Tian, J., Ma, L., \& Yu, W. (2011a). Ant colony optimization for waveletbased image interpolation using a three-component exponential mixture model. Expert Systems With Applications, 38, 1251412520.

Tian, J., Yu, W., Chen, L., \& Ma, L. (2011b). Image edge detection using variation-adaptive ant colony optimization. Transactions on Computational Collective Intelligence V. Berlin: Springer.

Torresani, L., Kolmogorov, V., \& Rother, C. (2008). Feature correspondence via graph matching: Models and global optimization. Computer Vision-ECCV 2008. Berlin: Springer.

Trucco, E. (1995). Geometric invariance in computer vision. AI Communications, 8, 193-194.

Tsai, Y.-H. R., Cheng, L.-T., Osher, S., \& Zhao, H.-K. (2003). Fast sweeping algorithms for a class of Hamilton-Jacobi equations. SIAM Journal on Numerical Analysis, 41, 673-694.

Tsitsiklis, J. N. (1995). Efficient algorithms for globally optimal trajectories. IEEE Transactions on Automatic Control, 40, 1528-1538.

Veltkamp, R. C. (2001). Shape matching: Similarity measures and algorithms. In: SMI 2001 International Conference on Shape Modeling and Applications (pp. 188-197).

Veltkamp, R. C., \& Hagedoorn, M. (2001). State of the art in shape matching. London: Springer.

Wang, S., Wang, Y., Jin, M., et al. (2007). Conformal geometry and its applications on $3 \mathrm{~d}$ shape matching, recognition, and stitching. IEEE Transactions on Pattern Analysis and Machine Intelligence, 29, 1209-1220.
Wang, Y., \& Teoh, E. K. (2004). A novel 2D shape matching algorithm based on B-spline modeling. In: 2004 International Conference on Image Processing, ICIP 2004 (pp. 409-412).

Wang, Y., Teoh, E. K., \& Shen, D. (2004). Lane detection and tracking using B-Snake. Image and Vision Computing, 22, 269-280.

Wang, Y., Teoh, E. K., \& Shen, D. (2001). Structure-adaptive B-snake for segmenting complex objects. In: Proceedings 2001 International Conference On Image Processing (pp. 769-772).

Weiss, I. (1993). Noise-resistant invariants of curves. IEEE Transactions on Pattern Analysis and Machine Intelligence, 15, 943-948.

White, R., Kamath, C., \& Newsam, S. (2004). Matching Shapes Using Local Descriptors. United States. Department of Energy.

Xingfang, Y., Yumei, H., \& Yan, L. (2010). An improved SUSAN corner detection algorithm based on adaptive threshold. In IEEE 2010 2nd International Conference on Signal Processing Systems (ICSPS, Vol. 2).

Xu, C., \& Duan, H. (2010). Artificial bee colony (ABC) optimized edge potential function (EPF) approach to target recognition for low-altitude aircraft. Pattern Recognition Letters, 31, 1759-1772.

Xu, C., Liu, J., \& Tang, X. (2009). 2D shape matching by contour flexibility. IEEE Transactions on Pattern Analysis and Machine Intelligence, 31, 180-186.

$\mathrm{Xu}$, J. (2008). Shape matching using morphological structural shape components. In: 15th IEEE International Conference on Image Processing, 2008. ICIP 2008 (pp. 2596-2599).

$\mathrm{Xu}, \mathrm{S}$., Han, L., \& Zhang, L. (2006). An algorithm to edge detection based on SUSAN filter and embedded confidence. In: 6th International Conference on Intelligent Systems Design and Applications 2006 (ISDA'06) (pp. 720-723).

Xu, Y., Wang, B., Liu, W., \& Bai, X. (2010). Skeleton graph matching based on critical points using path similarity. Computer VisionACCV 2009. Berlin: Springer.

Yang, Y-L., Lai, Y-K., Hu, S-M., \& Pottmann, H. (2006). Robust principal curvatures on multiple scales. In: Symposium on Geometry Processing (pp. 223-226).

Yu, B., Guo, L., Zhao, T., \& Qian, X. (2010). A curve matching algorithm based on Freeman Chain Code. In: Intell. Comput. Intell. Syst. (pp. 669-672).

Zahn, C. T., \& Roskies, R. Z. (1972). Fourier descriptors for plane closed curves. IEEE Transactions on Computers, 100, 269-281.

Zeng, J., \& Li, D. (2011). SUSAN edge detection method for color image. Jisuanji Gongcheng yu Yingyong, 47, 194-196.

Zhang, D., \& Lu, G. (2004). Review of shape representation and description techniques. Pattern Recognition, 37, 1-19.

Zhang, S., \& Ma, K-K. (2000). A novel shape matching method using biological sequence dynamic alignment. In: 2000 IEEE International Conference on Multimedia and Expo (ICME) (pp. 343-346).

Zhao, H. (2005). A fast sweeping method for eikonal equations. Mathematics of Computation, 74, 603-627.

Zhou, D., et al. (2011). Hybrid corner detection algorithm for brain magnetic resonance image registration. In: IEEE - 2011 4th International Conference on Biomedical Engineering and Informatics (BMEI, Vol. 1).

Zisserman, A., Forsyth, D., Mundy, J., et al. (1995). 3D object recognition using invariance. Artificial Intelligence, 78, 238-239. 